\title{
Los suelos de Casapalma (Valle del Guadalhorce, Málaga). Análisis edafogeográfico aplicado a la ordenación del territorio
}

\section{Soils of Casapalma (Guadalhorce Valley, Málaga). Edaphogeographical analysis applied to the land management}

\author{
Jesús Rodrigo Comino, Emilio Ferre Bueno y José María Senciales ${ }^{1}$
}

\section{INTRODUCCIÓN}

El suelo es un recurso indispensable para el hombre y el funcionamiento de los ecosistemas. Así lo reconocen organismos internacionales de alto grado de excelencia y tradición científica como FAO (Food and Agriculture Organization of the United Nations) y el USDA (United States Department of Agriculture). En relación a la capa edáfica, encontrar una definición en la actualidad que verse sobre este elemento del medio es relativamente sencillo; no obstante, la clave de su importancia recae, no ya en que sea una parte con un funcionamiento propio dentro del ecosistema natural y antropizado, sino en ser un actor principal que interactúa con gran parte de los elementos que lo rodea (Bryan, 2000; Poesen et al., 2003; Ruiz Sinoga et al., 2010; Cerdà et al., 2013). Por consiguiente, resulta indispensable considerar en la planificación territo-

\footnotetext{
${ }^{1}$ Los tres pertenecen a la Universidad de Málaga (España), Departamento de Geografía, Área de Geografía Física, Campus de Teatinos s/n. 29071, Málaga, España, correo: geo.jrc@gmail.com, teléfono: 637937558. Además, el primero de ellos pertenece a la Universidad de Trier (Alemania), Departamento de Geografía Física, Campus II, Behringstraße, 54296, Trier (Alemania), correo: s6jerodr@uni-trier.de
} 
rial el recurso suelo (Picarreta et al., 2006; Martínez-Casasnovas et al., 2010; Navarro et al., 2012) para minimizar el impacto del sellado, la erosión, la contaminación, el descenso de la fertilidad y del número de sus funciones, la destrucción del paisaje, el aumento de las temperaturas (a nivel microclimático), las pérdidas de retención de agua e incremento de la escorrentía, la pérdida de secuestro de carbono y de biodiversidad (Van Oost et al., 2007; Hernández Santana et al., 2010; Quinton et al., 2010; Porta y Poch, 2011).

De esta forma, para una adecuada ordenación del territorio, teniendo en cuenta el manto edáfico, cabe la posibilidad de aplicar métodos mixtos de análisis espacial desde la Agronomía, la Edafología, la Ecología o la Edafogeografía (Ferreras y Fidalgo, 1991). Algunas fuentes y métodos son comunes a estas ciencias. Así, el análisis edafogeográfico ${ }^{2}$ consiste en que, una vez clasificado el manto edáfico, se replantee la cartografía, se establezcan agrupaciones que permitan trabajar a muy diversas escalas y se abstraiga la sistemática territorial de los suelos para un diagnóstico final concreto (Rodrigo Comino y Senciales, 2013; Rodrigo Comino, 2014). Finalizados estos pasos, es imprescindible tener claro el campo de aplicación de los estudios a realizar, ya que de él dependerá, en principio, el tipo de clasificación y, posteriormente, los campos de la base de datos analítica y cartográfica con la que debería trabajarse (Boardman et al., 2003; Auerswald et al., 2009; Cerdan et al., 2010; Brevik et al., 2015).

Partiendo de esta base teórica, la aplicación final de este estudio será la Ordenación del Territorio como expresión final más afín de la Geografía de los Suelos (Dent y Young, 1981; Ferreras y Fidalgo, 1991; Zinck, 2012; Rodrigo Comino y Senciales, 2013; Rodrigo Comino, Ferre y Senciales, 2014; Rodrigo Comino, Senciales y Ferre, 2014; Rodrigo Comino, 2014).

La primera fuente que estudió los tipos de mantos edáficos del medio mediterráneo de forma sistemática en la península Ibérica (por la información, la escala y la precisión de los análisis) es la cartografía del Proyecto LUCDEME (Proyecto de Lucha contra la Desertificación en el Mediterráneo en 1981). Dichos mapas surgen como iniciativa del gobierno español tras las recomendaciones del Plan de Acción contra la Desertificación de las Naciones Unidas (DESCOM) de Nairobi en 1977 para: «promover los estudios y trabajos que

${ }^{2}$ La ignorancia generalizada de este término en España, pero muy común en países como Alemania (Gebhardt et al., 2012), obliga a enfatizar en que existen estudios con relevancia científica internacional en dicha línea de investigación. El término «Edafogeografía» fue acuñado por primera vez en una obra monográfica española en el título principal por Ferreras y Fidalgo (1991). 
permitieran conocer la situación real de las áreas afectadas y sus particularidades; cómo influyen los diferentes factores que dan lugar a los procesos relacionados con el fenómeno de la desertificación, y así facilitar el diseño de las medidas de acción adecuadas dentro de las premisas del desarrollo sostenible» (www.magrama.gob.es). El espacio que abarca se encuentra dentro de la región mediterránea de la península ibérica y en una primera fase acogía 32.622 $\mathrm{km}^{2}$, englobando las provincias de Almería y Murcia y parte de la de Granada. A partir de 1990, se amplía este territorio a la mayoría de la vertiente mediterránea española clasificando diferentes parámetros en función de distintas «áreas temáticas» (suelos, zoología, erosión hídrica, erosión eólica...). Entre estos trabajos, destacan los de ámbito edafológico, donde se realizaron 132 hojas de mapas de los tipos de suelos a escala 1:100000, con la metodología de FAO desarrollada en 1988, como las hojas 1:50000 de parte de la provincia de Málaga.

Se pueden considerar, concretamente para el caso de la provincia de Málaga, que la cartografía edáfica y los análisis de laboratorio del proyecto LUCDEME fueron la primera base de datos sistemática de su medio físico y humano, que surtió de una información elemental a los científicos que quisieran utilizar conocimientos edafológicos para sus estudios sobre el territorio. En el año 2000, se digitalizó en formato SIG, pero no se actualizó a la clasificación de FAO de 1998. Además, es importante añadir que, pese a ser una base de datos novedosa en el territorio de la provincia de Málaga, no abarcó todo su término administrativo. Otros problemas también detectados fueron: i) las numerosas confusiones en la identificación de los tipos edáficos; ii) algunos análisis no coincidían con el tipo de suelo identificado; iii) los límites, sea por la escala de trabajo o por la falta de coordinación, tampoco eran correctos, cerrándose algunos en una simple línea recta (Rodrigo Comino, 2014).

A partir de este hecho, se convirtió en una tarea prioritaria revisar todas estas fuentes antes de realizar cada estudio y aplicarlo a realidades concretas a menor escala, como la ladera. Como es bien sabido, la variabilidad de parámetros del suelo como la materia orgánica, la textura, el $\mathrm{pH}$, la relación $\mathrm{C} / \mathrm{N}$, la concentración de carbonatos o los procesos erosivos entre otros, son muy elevadas en los suelos naturales, pero más aún si cabe, en los alterados por las actividades agrícolas (Kosmas et al., 1997; Matarechera, 2009; Prasuhn, 2011; Arnáez et al., 2012; Rodrigo Comino et al., 2015; Rodrigo Comino et al., 2016). De esta forma, se hace indispensable conocer su estado físico inicial y aptitud química frente a las actividades que dependen de estos territorios ya sea utilizando análisis de suelos, elaborando perfiles o llevando a cabo experimentos in situ (Arnáez et al., 2007; IUSS-WRB, 2007; Raclot et al., 2009; 
Wang et al., 2013; Prodoscimi et al., 2016). Así, la extrapolación de datos a partir de perfiles de suelos colindantes de esta cartografía del LUCDEME no se puede considerar del todo apropiada, sin embargo, todavía no hay estudios a escala más reducida que digan lo contrario.

Para disciplinas como la Agronomía aplicada, la Edafología o la Ingeniería Civil el suelo es un complejo que puede estudiarse según unas propiedades químicas y físicas concretas dentro de formularios que provienen de laboratorios especializados, generalmente a escalas 1:10, 1:100 o 1:1000 (Chacón Montero et al., 2004). Sin embargo, desde el punto de vista de la Geografía de los Suelos (Rodrigo Comino y Senciales, 2013) o Geopedología (Zinck, 2012), la generación de una cartografía a diversa escala que «territorialice» dichas propiedades bajo unas condiciones geomorfológicas, biogeográficas, climáticas y antrópicas concretas se convierte en una herramienta muy útil para ordenar el territorio. Numerosos ejemplos se pueden encontrar en trabajos aplicados de investigación llevados a cabo para evaluar las políticas de gestión del territorio (Picarretta et al., 2006; Martínez-Casasnovas et al., 2010), los procesos de erosión (Poesen et al., 2003; Ruiz Sinoga et al., 2009; Paroissien et al., 2010) o la cartografía a «downscaling» con SIG (Hengl, 2006; Goodchild, 2011; Rukhovich et al., 2011; Wang et al., 2013; Galacho y Arrebola, 2013).

De esta forma, como objetivos para este trabajo se pretende: i) aumentar la escala desde 1:50000 a 1:25000 para poder reelaborar las bases de datos existentes; ii) revisar y adaptar la cartografía a través de una metodología combinada enunciada por el ITC (International Institute for Geo-Information Science and Earth Observation, Enschede, Países Bajos) denominada como «mapa de unidades de diagnóstico»; iii) aplicar técnicas de fotoedafología y elaboración de perfiles para elaborar un mapa de unidades geomorfoedáficas con la capacidad de acoger información edafológica útil para la ordenación del territorio; iv) demostrar que el uso de información ligada a perfiles de suelos periféricos a la zona de estudio no es recomendable.

\section{ÁREA DE ESTUdio}

Se elabora el estudio dentro de un espacio integrado en el tramo medio del río Guadalhorce (provincia de Málaga) con una superficie de $18,72 \mathrm{~km}^{2}$, que coincide con la situación exacta de una ortofotografía a escala 1:18000 del vuelo de 1978. El «río del trigo», como es conocido este cauce, adquiere una complejidad en la disposición espacial de los materiales muy elevada, aunque se observan algunas directrices más o menos marcadas en algunos ejes de ple- 
gamientos triásicos (Sanz de Galdeano y López Garrido, 2013). Tiene un trazado E-W en su inicio y N-S y W-E a medida que se acerca a la costa, donde desemboca en una zona con gran contenido de arenas y aguas salinas, y dificultades para el drenaje. Posee un carácter eminentemente calcáreo y está controlado por numerosas presas (Ocaña y Larrubia, 1993).

Cerca de su tramo final, el área de estudio se enmarca en la unidad fisiográfica conocida como Hoya de Málaga en los términos administrativos de los municipios de Cártama y Pizarra, concretamente, en un área conocida como Casapalma (figura 1).

La unidad geomorfológica de la Hoya de Málaga queda delimitada en gran parte por el transcurso de su valle fluvial y existe un aprovechamiento agrícola de carácter intensivo, dados los suelos profundos y horizontes bien estructurados que se han formado (Natera, 2007; Rodrigo Comino, Senciales y Ferre, 2014).

Geomorfológicamente, los resultados de Rodrigo Comino et al., (2014) describieron el área de estudio de Casapalma como un valle fluvial cuyo fondo está compuesto de gravas, arenas y limos conformando pendientes suaves (3-8\%). A partir de aquí, se apreciaban una serie de escarpes irregulares de arcillas, margas y areniscas, entremezcladas con diferentes niveles de terrazas fluviales (consolidadas y no consolidadas). Dichas morfologías compuestas por arenas y margas, se pueden observar con diferentes grados de erosión, según la intensidad de dos factores incidentes: i) el manejo intensivo del suelo (agricultura, construcción...); ii) afección por las crecidas del cauce principal y sus afluentes. Uno de los rasgos más identificativos de este paisaje son las laderas en forma de glacis o superficies de erosión, con unos niveles de pendientes medios entre $8-21 \%$ y compuesto por arenas, margas, areniscas $y$, en algunos casos, arcillas. Esto hace de estos espacios, áreas de cultivos aprovechables y apreciadas, a la vez que vulnerables por su alto grado de erodibilidad. Por último, conforme nos alejamos del cauce principal se puede observar la aparición de un tipo de morfología totalmente distinta y que corresponde al conjunto geológico del maláguide, perteneciente a las unidades del Sistema Bético. En este paisaje montuoso en forma de lomo de elefante, las pendientes llegan a alcanzar intervalos de entre $21-31 \%$ y $46-76 \%$. Los materiales que lo conforman son muy variados, notándose litologías metamórficas datadas en el Precámbrico y el Paleozoico como las grauwacas, las pizarras o las filitas, o por el contrario, de tipo sedimentario como las margas, las calizas alabeadas o las dolomías. Cabe destacar que estas geoformas dan lugar, a menudo, a taludes de derrubios y escarpes irregulares también con elevadas pendientes $(>21 \%)$, que pueden generar riesgos de derrumba- 


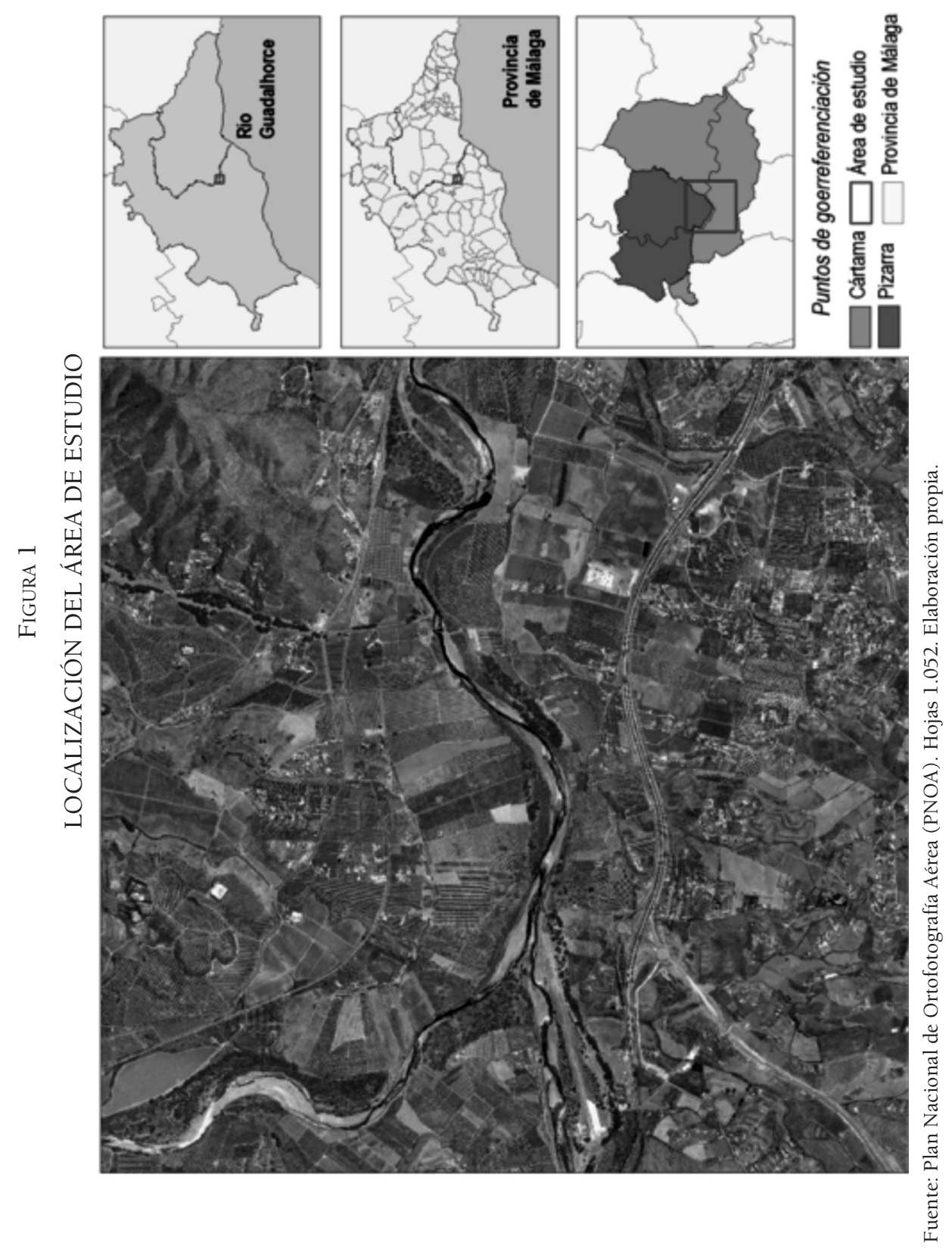

Estudios Geográficos, Vol. LXXVII, 280, pp. 275-310, enero-junio 2016

ISSN: 0014-1496, eISSN: 1988-8546, doi: 10.3989/estgeogr.201610 
miento y entarquinamiento con el material transportado a los cauces tributarios del río principal.

Según Larrubia (1994) el río Guadalhorce y su territorio «conjugan una realidad que mezcla actividades relacionadas directamente con su medio natural y otras de mera explotación económica ligadas a la agricultura o la ganadería». Antes de poner en funcionamiento los regadíos con los cítricos en dicha zona sobre las terrazas y los glacis, los usos más extendidos eran los cultivos de secano (olivar y herbáceos) que ocupaban casi un 75\% del total (y ahora relegados a los relieves del conjunto maláguide). Las nuevas reconversiones al sector servicios y el incremento de la accesibilidad por la mejora de las vías de comunicación y su unión con la costa han servido para la inversión en proyectos para el regadío, la mayor productividad en otros subsectores asociados y la conectividad del municipio de Cártama. Por una parte, se comprueba cómo los cultivos leñosos regados, fundamentalmente los cítricos (330,4 ha), y los cultivos regados herbáceos ocupan ahora más de un $40 \%$ del territorio (Rodrigo Comino, Senciales y Ferre, 2014). Los nuevos complejos de urbanizaciones agrícolas o residenciales y los procesos de periurbanización de las nuevas «postmetrópolis» (Soja, 2001) han salido beneficiados por estos cambios, perdiéndose casi 600 ha. en el total cultivado (Rodrigo Comino, Senciales y Ferre, 2014).

Por otra parte, pese a que la ETP media anual es elevada (900-950 mm) y las precipitaciones no equilibran la balanza de pérdida de agua del suelo (600$700 \mathrm{~mm}$ ), la cercanía a un ecosistema fluvial salva dichas diferencias por la disposición del recurso hídrico directamente del cauce. Las especies vegetales están condicionadas por las elevadas temperaturas $\left(15-17^{\circ} \mathrm{C}\right.$, que alcanzan más de $19^{\circ} \mathrm{C}$ en la media de las máximas), lo cual determina la presencia de especies adaptadas al estrés hídrico conforme se incrementa la distancia al lecho principal del río (García Marín, 2009). Secundariamente, queda condicionado el control del agua que afecta al crecimiento y a las características o propiedades que influyen sobre los procesos que incluyen la estabilidad y degradación edáfica (Lado et al., 2004; Ruiz Sinoga et al., 2010).

\section{MATERIAL Y MÉTODOS}

Se establecen unas líneas de investigación basadas en una metodología para el estudio de los suelos desde el punto de vista teórico y práctico de la Edafogeografía o Geografía de los Suelos (Rodrigo Comino y Senciales, 2013; Rodrigo Comino, 2014). 
La siguiente propuesta está soportada en aplicaciones SIG y en observaciones de campo. En primer lugar, se reescalará la información de los mapas (desde 1:50000 a 1:10000) y se cartografiará geomorfológicamente el área de estudio (Van Zuidam y Van Zuidam Cancelado, 1979). Esto se llevará a cabo superponiendo (con la herramienta de unión de capas en formato vectorial) mapas temáticos de morfometría, asociaciones litológicas, morfologías y sistemas de paisaje (Panizza et al., 1987; Panizza, 2005; Rodrigo Comino, Ferre y Senciales, 2014). Dicha cartografía fue elaborada a partir de trabajo de campo y fotointerpretación, siguiendo las mismas pautas que Zinck (2012). Según estos autores, de esta forma se puede evaluar y validar la cartografía gracias a técnicas genéticas, paisajísticas y paramétricas. Así, áreas que no son afines geomorfológicamente, no lo podrán ser con una gran probabilidad edafológicamente. Estas técnicas a partir del uso del SIG permiten trabajar a varios niveles de detalle y extrapolar patrones para otros territorios, aunque deba ajustarse la escala y las variables según el medio (Hengl, 2006; Goodchild, 2011). Por otra parte, no ocurre lo mismo con los límites de mantos edáficos distintos o determinados aspectos particulares (Nieves y Torcal 1983), para los que sería más útil emplear la elaboración de «catenas» o «toposecuencias» (Yaalon, 1975; Birkeland, 1984).

El mapa de suelos elaborado por el proyecto LUCDEME que abarca parte de la provincia de Málaga contiene numerosos errores de identificación, delimitación y elaboración como se ha mencionado anteriormente (Rodrigo Comino, 2014). Para poder revisar las fuentes y elaborar el mapa de unidades geomorfoedáficas (útil para la ordenación del territorio desde un punto de vista edafogeográfico), se recurrió a una secuencia metodológica creada a partir de los trabajos de Van Zuidam y Van Zuidam Cancelado (1979), Dent y Young (1981), Porta y López-Acevedo (2005), Zinck (2012), Galacho y Arrebola (2013), Rodrigo Comino, Ferre y Senciales (2014) y Rodrigo Comino, Senciales y Ferre (2014).

En primer lugar, se realizaron una serie de perfiles de suelos en las áreas donde no casaba la fotografía aérea o las características geomorfológicas de base, con el tipo de suelo propuesto por el mapa edafológico a escala 1:50000, según el método del IUSS Working Group WRB (2006a, 2006b y 2007). Esta actividad es definida por Birkeland (1984) como «la observación vertical de todos los horizontes del manto edáfico desde el material parental» y según Duchaufour (1987), útiles para evaluar las variabilidades elevadas de sus tipos a través de las formas del relieve. Para su estudio, se requiere cada vez más el uso de nuevas tecnologías de procesado de la información y la participación de científicos más transversales (Ibáñez et al., 1990; Porta y López-Acevedo, 
2005). Para su elaboración, también se ha seguido un material sintetizado de los autores Viguera et al. (2004), y Porta y López-Acevedo (2005). Como fuentes auxiliares, además, se visualizaron los nuevos recursos a disposición de los investigadores en internet de Cartografía Digital de Suelos o CDS (www.fao.org/soils-portal/levantamiento-de-suelos/es/), eSOTER (www.esoter. net/), Global-Soil (www.globalsoilmap.net/) y las aplicaciones para Google Earth de mapas digitalizados de la Comisión Europea (ec.europa.eu/environ ment/soil/index_en.htm).

A partir de aquí, se digitalizaron las nuevas asociaciones y consociaciones registradas con el software ArcGis 10.1 y sus herramientas de edición de capas vectoriales. El resultado es una información espacial precisa de cada asociación y consociación revisada con el mapa de suelos del Proyecto LUCDEME y los nuevos recursos de internet de georeferenciación. Todo esto queda asociado a unas bases de datos con las características geomorfológicas y antrópicas sobre las que se activa la edafogénesis y se desarrollan los horizontes, con una escala más precisa de trabajo.

Por último, tras la revisión de las unidades geomorfoedáficas, para poder observar las propiedades físico-químicas de los suelos con objeto de distinguir áreas para según qué tipo de usos, se añadió información de laboratorio desde perfiles periféricos. Para dicho fin, se utilizaron los resultados del proyecto LUCDEME (Hoja 1052: Aguilar Ruiz et al., 1994), seleccionándose suelos semejantes por su tipología y situación geográfica, a través de un procesado estadístico de la información con el software SPSS 21. Por último, se adjunta a través de una tabla todos los datos a la capa vectorial final del SIG.

\section{RESULTADOS}

\section{Elaboración del mapa de unidades de diagnóstico}

Para la elaboración del mapa de las «unidades de diagnóstico» (Van Zuidam y Van Zuidam Cancelado, 1979), se ha considerado una serie de variables básicas acordes al medio mediterráneo, donde cada categoría representará un nivel de estudio diferenciado por el alcance de su nivel de detalle o abstracción en la escala de análisis: morfometría, asociaciones litológicas, morfologías y sistemas de paisaje. El mapa de base resultante llamado «unidades de diagnóstico» se presenta en la figura 2 con una tabla adjunta (1) explicativa de cada zonificación, las características inventariadas y fotointerpretadas. 

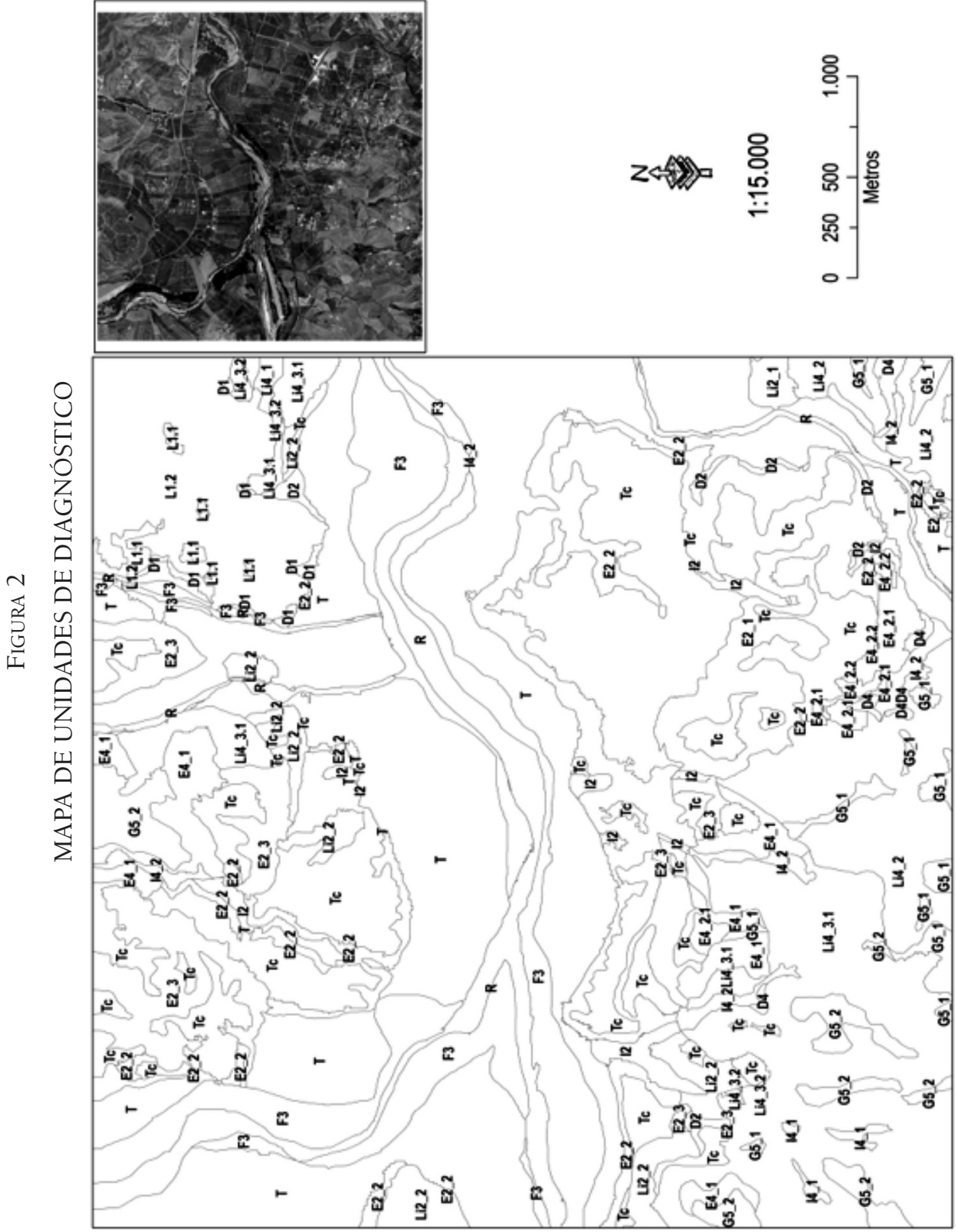

Estudios Geográficos, Vol. LXXVII, 280, pp. 275-310, enero-junio 2016

ISSN: 0014-1496, eISSN: 1988-8546, doi: 10.3989/estgeogr.201610 
TABLA 1

DESCRIPCIÓN DE LAS CARACTERÍSTICAS GEOMOROFLÓGICAS DE LAS UNIDADES DE DIAGNÓSTICO

\begin{tabular}{|c|c|c|c|c|c|}
\hline ID & Morfología & Agrupación litológica & $\begin{array}{l}\text { Pendien- } \\
\text { tes (\%) }\end{array}$ & $\mathrm{Ha}$ & $\begin{array}{l}\% \text { sobre } \\
\text { el total }\end{array}$ \\
\hline $\mathrm{Dl}$ & Talud de derrubios sobre materiales maláguides & Grauwacas, calcofilitas y calizas alabeadas & $21-31$ & 4,29 & 0,23 \\
\hline D2 & $\begin{array}{l}\text { Talud de derrubios sobre terrazas fluviales } \\
\text { erosionadas }\end{array}$ & Conglomerados, gravas, arenas y limos & $16-21$ & 6,69 & 0,36 \\
\hline D4 & Talud de derrubios con laderas irregulares & Gravas, arenas, limos y arcillas & $16-21$ & 7,89 & 0,42 \\
\hline E2_1 & Escarpes y terrazas fluviales erosionadas & Arenas y margas & $16-21$ & 74,4 & 3,97 \\
\hline E2_2 & Escarpes y terrazas fluviales erosionadas & $\begin{array}{l}\text { Conglomerados, gravas, arenas, limos } \\
\text { y margas }\end{array}$ & $16-21$ & 100,03 & 5,34 \\
\hline $\mathrm{E} 2 \_3$ & Escarpes y terrazas fluviales erosionadas & Arcillas, margas y areniscas & $16-21$ & 71,84 & 3,84 \\
\hline E4_l & Escarpes con laderas irregulares & Arcillas, margas y areniscas & $16-21$ & 45,24 & 2,42 \\
\hline E4_2.1 & Escarpes con laderas irregulares (<31\%) & Conglomerados, gravas, arenas y limos & $16-21$ & 16,37 & 0,87 \\
\hline E4_2.2 & Escarpes con laderas irregulares (>31\%) & Conglomerados, gravas, arenas y arcillas & $31-46$ & 3,04 & 0,16 \\
\hline F3 & Fondo del río y cauce arenoso & Gravas, arenas y limos & 8-16 & 158,28 & 8,45 \\
\hline G5_l & Glacis y superficies de erosión & Arenas y margas & $16-21$ & 22,21 & 1,19 \\
\hline G5_2 & Glacis y superficies de erosión & Arcillas, margas y areniscas & 8-16 & 37,78 & 2,02 \\
\hline I2 & Fondo de valle con terrazas erosionadas & Gravas, arenas, limos y arcillas & $16-21$ & 20,7 & 1,1 \\
\hline I4_1 & Fondo de valle con laderas irregulares & Arcillas, margas y areniscas & $16-21$ & 3,02 & 0,16 \\
\hline I4_2 & Fondo de valle con laderas irregulares & Gravas, arenas, limos y arcillas & $16-21$ & 15,61 & 0,83 \\
\hline Ll.1 & Montaña media de origen maláguide ( $<31 \%)$ & Montaña media de & $21-31$ & 6,69 & 0,36 \\
\hline $\mathrm{L} 1.2$ & Montaña media de origen maláguide (>31\%) & Grauwacas, calcofilitas y calizas alabeadas & $46-76$ & 101,37 & 5,41 \\
\hline Li2_1 & $\begin{array}{l}\text { Terrazas fluviales erosionadas sobre laderas } \\
\text { irregulares }\end{array}$ & Arenas y margas & $21-31$ & 5,27 & 0,28 \\
\hline Li2_2 & $\begin{array}{l}\text { Terrazas fluviales erosionadas sobre laderas } \\
\text { irregulares }\end{array}$ & Arcillas, margas y areniscas & $21-31$ & 40,73 & 2,17 \\
\hline Li4_1 & Laderas irregulares sobre materiales maláguides & Grauwacas, calcofilitas y calizas alabeadas & $21-31$ & 2,57 & 0,14 \\
\hline Li4_2 & $\begin{array}{l}\text { Terrazas fluviales erosionadas sobre laderas } \\
\text { irregulares }\end{array}$ & Arenas y margas & $16-21$ & 99,48 & 5,31 \\
\hline Li4_3.1 & Escarpes con laderas irregulares (<31\%) & Arcillas, margas y areniscas & $16-21$ & 186,66 & 9,97 \\
\hline Li4_3.2 & Escarpes con laderas irregulares ( $>31 \%)$ & Arcillas, margas y areniscas & $46-76$ & 3,02 & 0,16 \\
\hline $\mathrm{R}$ & Cauce ordinario & Gravas, arenas y limos & 3-8 & 81,41 & 4,35 \\
\hline $\mathrm{T}$ & Terrazas fluviales no consolidadas & Gravas, arenas, limos y arcillas & $0-3$ & 469,41 & 25,06 \\
\hline Tc & Terrazas fluviales consolidadas & Conglomerados, gravas, arenas y limos & $8-16$ & 288,91 & 15,43 \\
\hline
\end{tabular}




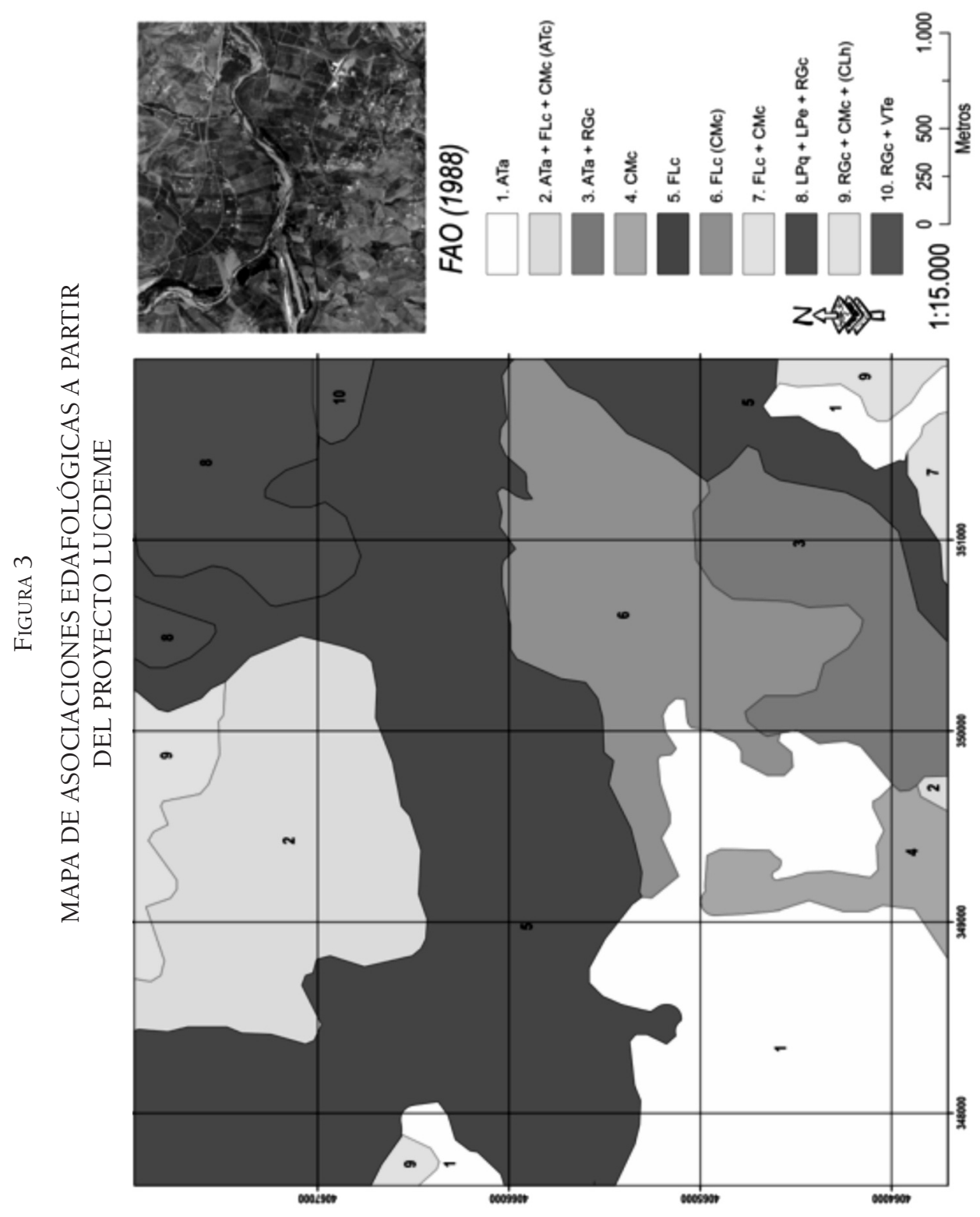

Estudios Geográficos, Vol. LXXVII, 280, pp. 275-310, enero-junio 2016

ISSN: 0014-1496, eISSN: 1988-8546, doi: 10.3989/estgeogr.201610 


\section{Elaboración del mapa de unidades geomorfoedáficas}

El siguiente paso, consta de la realización de un análisis de las fuentes edafológicas previas (figura 3 y tabla 2) activando las capas en formato vectorial de los suelos identificados por el proyecto LUCDEME (con la leyenda antigua del mapa de FAO de 1988) en el SIG.

TABLA 2

DESCRIPCIÓN DE LA LEYENDA DEL MAPA DE LA FIGURA 4

\begin{tabular}{cccccc}
\hline Abreviatura & Ata & ATc & CLh & CMc & FLk \\
\cline { 1 - 4 } $\begin{array}{c}\text { Nombre } \\
\text { completo }\end{array}$ & $\begin{array}{c}\text { Antrosol } \\
\text { árico }\end{array}$ & $\begin{array}{c}\text { Antrosol } \\
\text { cumúilico }\end{array}$ & $\begin{array}{c}\text { Calcisol } \\
\text { háplico }\end{array}$ & $\begin{array}{c}\text { Cambisol } \\
\text { calcárico }\end{array}$ & $\begin{array}{c}\text { Fluvisol } \\
\text { cálcico }\end{array}$ \\
\hline Abreviatura & LPq & LPe & RGc & VTe & \\
\cline { 1 - 4 } $\begin{array}{c}\text { Nombre } \\
\text { completo }\end{array}$ & $\begin{array}{c}\text { Leptosol } \\
\text { lítico }\end{array}$ & $\begin{array}{c}\text { Leptosol } \\
\text { éutrico }\end{array}$ & $\begin{array}{c}\text { Regosol } \\
\text { calcárico }\end{array}$ & $\begin{array}{c}\text { Vertisol } \\
\text { éutrico }\end{array}$ & \\
\hline
\end{tabular}

En esta cartografía, se aprecian las diferencias entre el paisaje subyacente de la fotografía aérea y los trazos de los límites de los tipos de asociaciones edáficas cartografiadas. Se observa cómo no coinciden elementos claves, según Dent y Young (1981), como son los usos y coberturas de vegetación, los tipos de tierras, las formas de relieve, las pendientes, los drenajes constructivos y destructivos de los cauces, la estratigrafía o, simplemente, los colores.

Tras examinar la fotografía aérea con detenimiento, las características geomorfológicas del territorio y la bibliografía, el paso siguiente es elaborar perfiles de suelos para corregir y aumentar la escala de trabajo. Se propone una serie de ejemplos localizados en el área de estudio a partir de la fotografía aérea (figura 4) y realizados en las áreas donde las características geomorfológicas del área de estudio no casaban con los suelos identificados (figuras 5, 6, 7 y 8 ). 
FIGURA 4

CROQUIS DE LA LOCALIZACIÓN DE LOS PERFILES REALIZADOS EN ÁREAS DUDOSAS

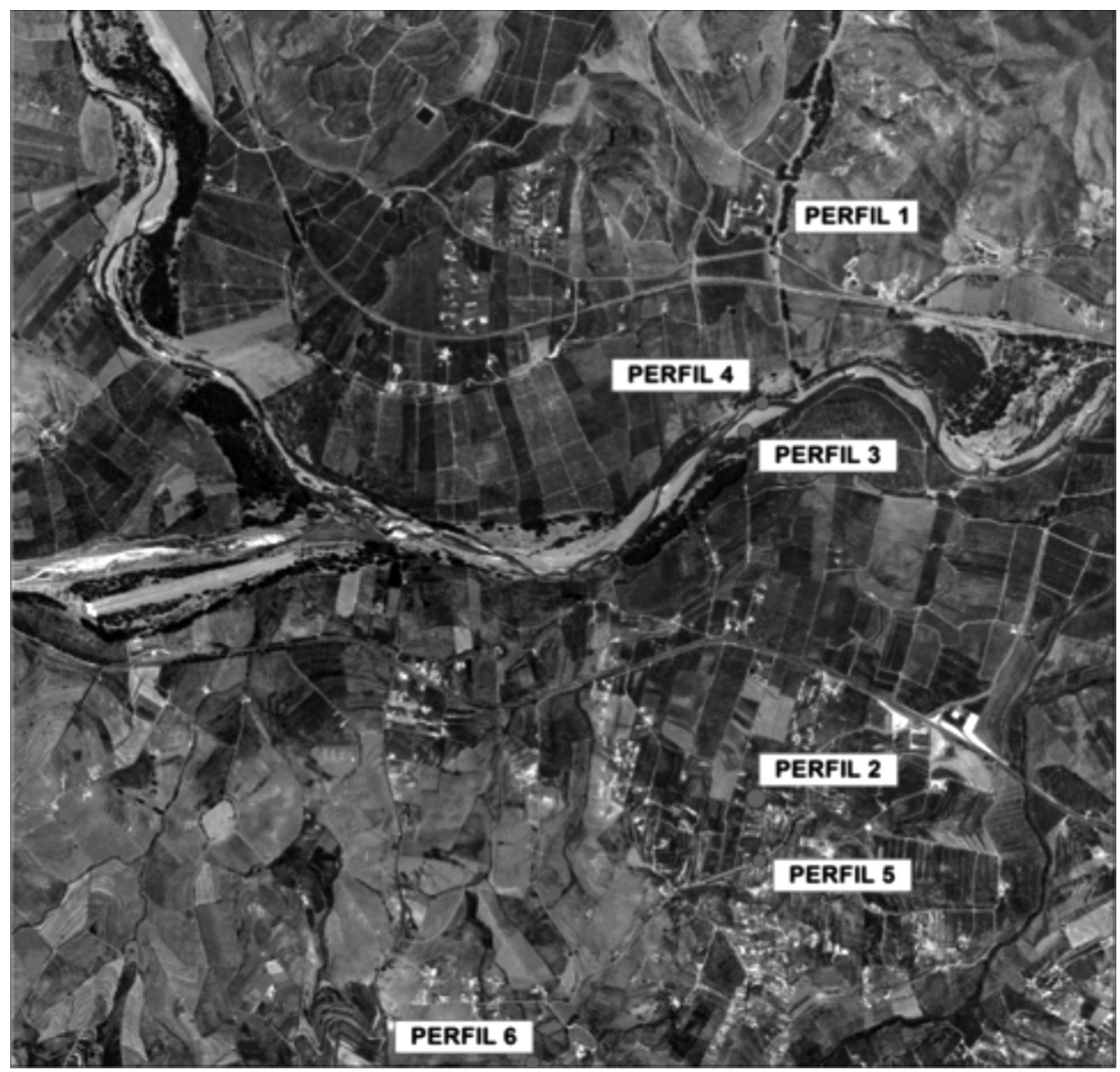

Estudios Geográficos, Vol. LXXVII, 280, pp. 275-310, enero-junio 2016

ISSN: 0014-1496, eISSN: 1988-8546, doi: 10.3989/estgeogr.201610 


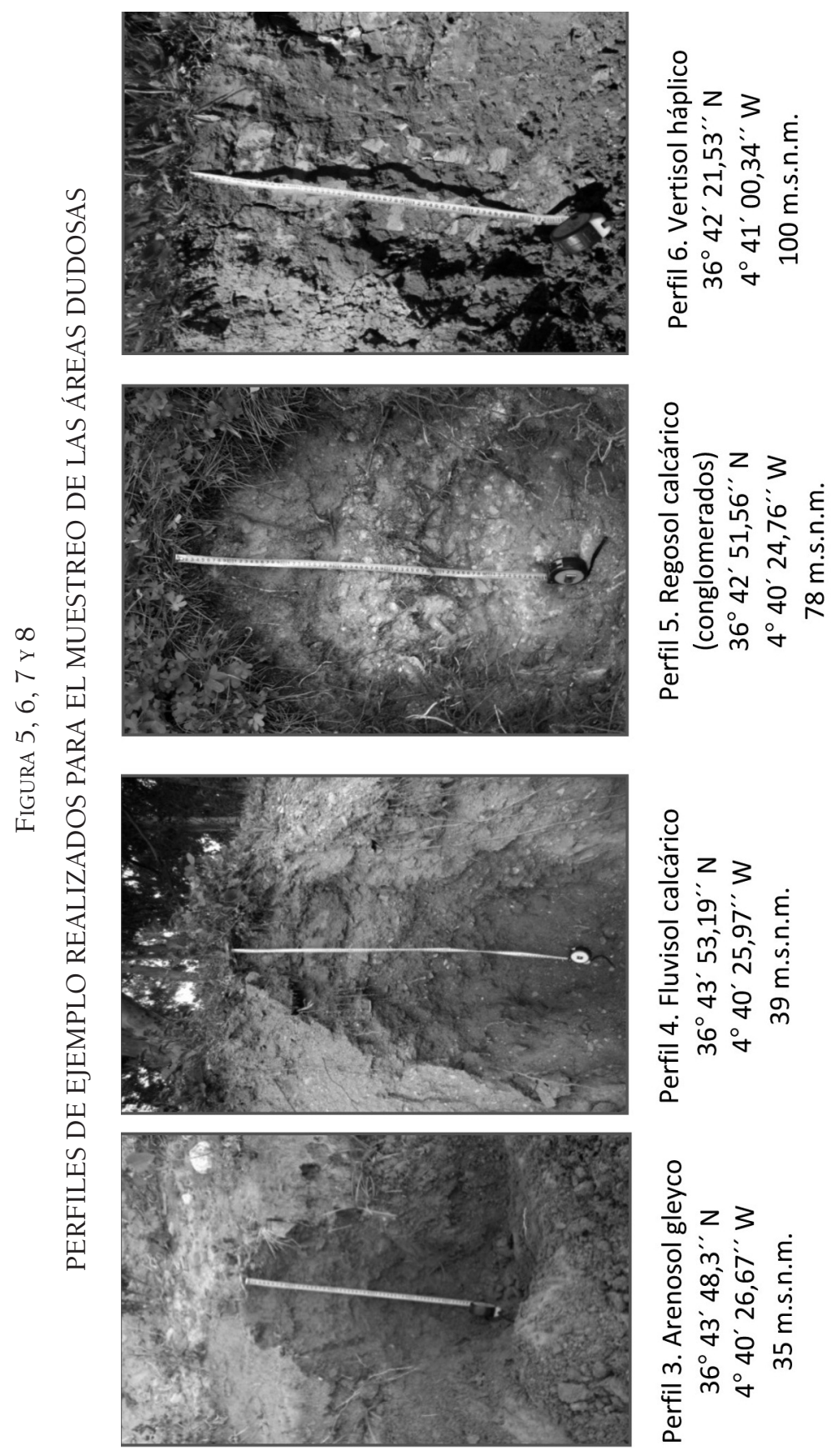

Estudios Geográficos, Vol. LXXVII, 280, pp. 275-310, enero-junio 2016 ISSN: 0014-1496, eISSN: 1988-8546, doi: 10.3989/estgeogr.201610 
A continuación, se presentan como ejemplos dos perfiles analizados durante el trabajo de campo ( 1 y 2). El perfil 1 corresponde a un Regosol calcárico en el mapa edafológico, pero al comprobar con ácido clorhídrico la reacción nula hasta una profundidad de 1 metro, se ha descartado este tipo de taxón. Con respecto al 2 (y ratificado con el 5), identificado como un Antrosol árico, tampoco al realizar el perfil se podría catalogar como tal porque no posee ni restos de artefactos o acciones de labranza profunda, ya que estas áreas simplemente habían sido antiguos cultivos de olivo o naranjos. Observando este espacio, el Vertisol es el tipo más adecuado, vistos los problemas de piping, agrietamiento de la superficie y cicatrices de despegue por el movimiento generado por las arcillas montmorilloníticas cartografiadas en el mapa geológico de la hoja 1052 (IGME, 1978) o Cambisoles con propiedades vérticas. Los perfiles realizados en 3 y 4 sirvieron para cerciorar la aparición de Fluvisoles calcáricos y Arenosoles gleycos estacionalmente afectados por el ascenso del nivel freático.

\section{Ejemplo 1: PERFIL 1. ALJAIMA:}

Clasificación: Regosol éutrico (FAOWRB, 2006)

Provincia: Málaga.

Situación: Sierra de Aljaima (Cártama) en la salida de la carretera "Dehesas altas".

Coordenadas: $36^{\circ} 44$ 13,89" (N) $4^{\circ} 40$ $23,33^{\prime \prime}(\mathrm{W})$.

Altitud: $72 \mathrm{~m}$.

Posición fisiográfica: Ladera convexa. Forma del terreno circundante:

Fuerte/socavado.

Pendiente: Escarpada (31-46\%).

Orientación: $330^{\circ} \mathrm{NWW}$.

Vegetación o uso: Cultivos semiabandonados de Olea europea y Prunus amygdalus, matorrales como Asphodelus albus, Chamaerops humilis, Asparagus officinnalis, Rosmarinus officinalis, Marrubium vulgare y herbáceas de tipo Festuca.

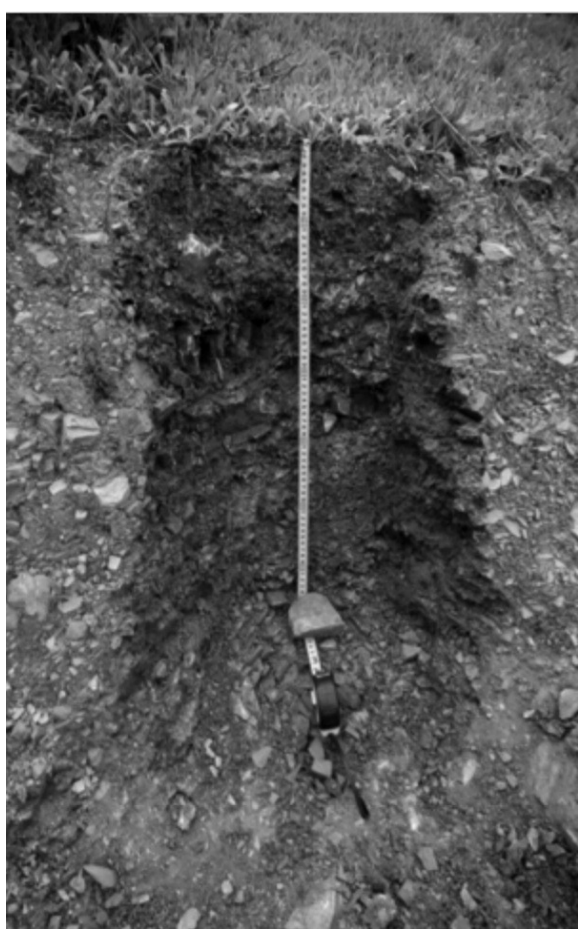


Material original: Pizarras saprolitizadas.

Drenaje: Excesivamente drenado.

Pedregosidad: Moderadamente pedregoso.

Afloramientos rocosos: Ninguno.

Erosión: Hídrica (laminar, surcos y cárcavas) e influencia humana (desmonte y aterrazamientos).

\begin{tabular}{lcc}
\hline Horizonte & $\begin{array}{c}\text { Profundidad } \\
(\mathrm{cm})\end{array}$ & Descripción \\
\hline
\end{tabular}

A1 0-13/21 Con color 7,5YR 5/3 en seco y (5YR 3/3) en húmedo. Límite ondulado y abrupto. Textura: Franco arenosa, estructura laminar y muy gruesa, desarrollada con una consistencia ligeramente dura en mojado, moderada en húmedo y ligeramente dura en seco y moderada en húmeda. Sin cutanes y rocosidad aparente. Pocas raíces finas, distribuidas vertical y horizontalmente. Los poros son intersticiales, son muy numerosos y frecuentes. Sin reacción carbonática.

A2 0-13/21-42 Con color 7,5YR 4/6 en seco y en húmedo. Límite claro y plano. Textura franco arenosa/arcillosa; estructura en bloques subangulares y muy fina, desarrollada con una consistencia plástica en seco y muy débil mojado el agregado. Aparecen cutanes de materia orgánica entre el límite del horizonte $A_{2}$ y $B$. Muy pocas raíces, muy finas y distribuidas de forma vertical. Por último, los poros son intersticiales y vesiculares, muy finos y poco frecuentes. Sin reacción carbonática.

B 20/50 Con color 10YR 4/4 en seco y en húmedo. Límite plano y abrupto. Textura franca; estructura en bloques angulares, moderada y gruesa, desarrollada con una consistencia dura en seco y ligeramente adherente y muy plástica en húmedo. Aparecen muy pocos cutanes de materia orgánica en las caras de los agregados entre el límite del horizonte A2 y B. Muy pocas raíces, muy finas y distribuidas de forma horizontal. Por último, los poros son planares y vesiculares, muy finos y frecuentes. Sin reacción carbonática.

C $\quad>50$ Pizarra saprolitizada con color 5YR 4/6, con estructura de carácter lítico, con ausencia de raíces, poros y reacción con el carbonato. Aparecen cutanes de arcillas (muestras de la fersialitización), de carácter diferenciado y horizontal. 


\section{Ejemplo 2. Perfil 2 sobre conglomerados (regosoles erróneos):}

Clasificación: Cambisol calcárico

Provincia: Málaga.

Situación: Camino de la Dehesa Alta (Cártama)

Coordenadas: $36^{\circ} 42$ 49,96" (N) $4^{\circ} 40$ $25,69^{\prime \prime}(\mathrm{W})$.

Altitud: 79 metros.

Posición fisiográfica: Terracetas en pendientes cóncavas.

Forma del terreno circundante:

Fuerte/socavado.

Pendiente: Escarpada (31-46\%).

Orientación: $300^{\circ} \mathrm{NW}$.

Vegetación o uso: Pinus halepensis,

Araucaria karoo, Oxalis pes-caprae

L. y Agave americana.

Material original: Arenas y margas.

Drenaje: Moderadamente bien drenado.

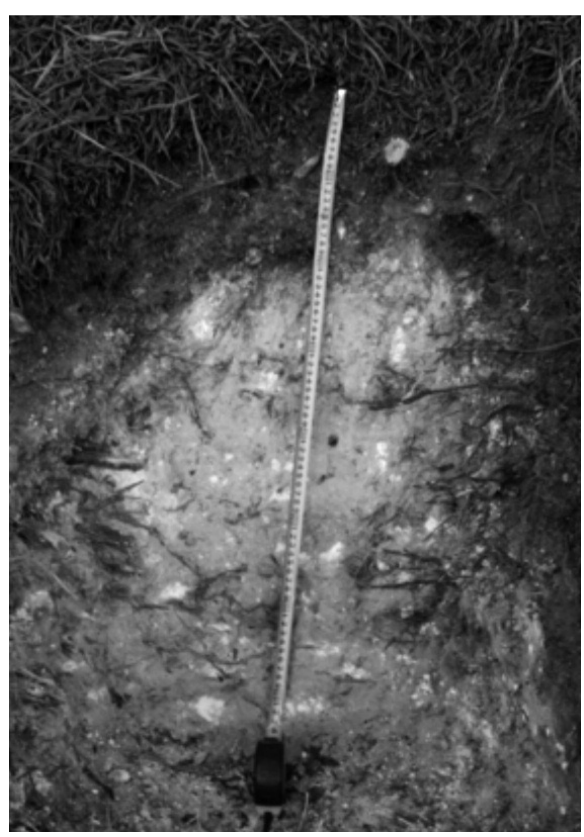

Pedregosidad: Moderadamente pedregoso.

Afloramientos rocosos: Ninguno.

Erosión: Hídrica (laminar, surcos y cárcavas) e influencia humana (arado, desmonte y aterrazamientos: «Terracetas»).

\begin{tabular}{|c|c|c|}
\hline Horizonte & $\begin{array}{l}\text { Profundidad } \\
\quad(\mathrm{cm})\end{array}$ & Descripción \\
\hline $\mathrm{AO}$ & $0-6$ & $\begin{array}{l}\text { Horizonte superficial compuesto por un mantillo com- } \\
\text { puesto por hojas y restos de corteza de las especies vegeta- } \\
\text { les que crecen sobre dicho territorio con origen antrópico } \\
\text { (pinos, araucarias o vinagretas). No se considerará parte del } \\
\text { análisis por no depender de procesos totalmente naturales } \\
\text { esta generación de materia orgánica. }\end{array}$ \\
\hline Bw & $6-18 / 20$ & $\begin{array}{l}\text { Con color 10YR } 4 / 4 \text { en seco y en húmedo } 10 \text { YR } 3 / 3 \text {. Límite } \\
\text { claro y ondulado. Textura franco arcillosa; estructura en } \\
\text { bloques granular y muy gruesa, desarrollada con una con- } \\
\text { sistencia ligeramente dura, no adherente y no plástica en } \\
\text { seco y muy friable mojado el agregado. No aparecen cuta- }\end{array}$ \\
\hline
\end{tabular}


C $>18 / 20$

nes, signo de la no presencia de procesos de iluviación de arcillas. Raíces muy abundantes, gruesas y distribuidas de forma horizontal. Por último, los poros son intersticiales y tubulares, de tipo mixto (finos, medianos,...). Reacción carbonática fuerte. Como rasgo biológico destacado, aparecen algunos insectos.

Con color 2,5Y 7/2 en seco y en húmedo 2,5Y 6/3. Límite plano y claro. Textura franco limosa; estructura lítica y prismática por la cementación de los carbonatos que dejan manchas en todo el horizonte coincidiendo con las áreas circundantes a las raíces (color: 2,5Y 6/6). También es de carácter fuertemente desarrollado y mediana, se desarrolla con una consistencia ligeramente dura en seco y ligeramente adherente y muy friable, plástica y adherente en húmedo. No aparecen cutanes de materia orgánica ni de arcillas, dando a entender la nula actividad en movimientos de iluviación-eluviación. Pocas raíces, gruesas y distribuidas de forma horizontal, las cuales alteran el material parental con procesos de degradación. Por último, los poros son planares y tubulares, de carácter mixto en su tamaño (desde muy finos a medianos) y muy pocos. Con una reacción carbonática extremadamente fuerte.

Partiendo de esta información, se elabora el mapa final de unidades geomorfoedáficas (figura 9). En la tabla 3 se adjunta una descripción de cada abreviatura utilizada en la cartagorafía de las asociaciones y consociaciones.

TABLA 3.

DESCRIPCIÓN DE LA LEYENDA DEL MAPA DE LA FIGURA 9

\begin{tabular}{ccccccc}
\hline Abreviatura & Ati & ATh & ARg & CLh & CMc & FLk \\
\hline $\begin{array}{c}\text { Nombre } \\
\text { completo }\end{array}$ & $\begin{array}{c}\text { Antrosol } \\
\text { irrágrico }\end{array}$ & $\begin{array}{c}\text { Antrosol } \\
\text { hórtico }\end{array}$ & $\begin{array}{c}\text { Arenosol } \\
\text { gléyico }\end{array}$ & $\begin{array}{c}\text { Calcisol } \\
\text { háplico }\end{array}$ & $\begin{array}{c}\text { Cambisol } \\
\text { calcárico }\end{array}$ & $\begin{array}{c}\text { Fluvisol } \\
\text { cálcico }\end{array}$ \\
\hline $\begin{array}{c}\text { Abreviatura } \\
\text { FLsq }\end{array}$ & LPq & LPe & RGc & RGe & VTh \\
\hline $\begin{array}{c}\text { Nombre } \\
\text { completo }\end{array}$ & $\begin{array}{c}\text { Fluvisol } \\
\text { subacuático }\end{array}$ & $\begin{array}{c}\text { Leptosol } \\
\text { lítico }\end{array}$ & $\begin{array}{c}\text { Leptosol } \\
\text { éutrico }\end{array}$ & $\begin{array}{c}\text { Regosol } \\
\text { calcárico }\end{array}$ & $\begin{array}{c}\text { Regosol } \\
\text { éutrico }\end{array}$ & $\begin{array}{c}\text { Vertisol } \\
\text { háplico }\end{array}$ \\
\hline
\end{tabular}




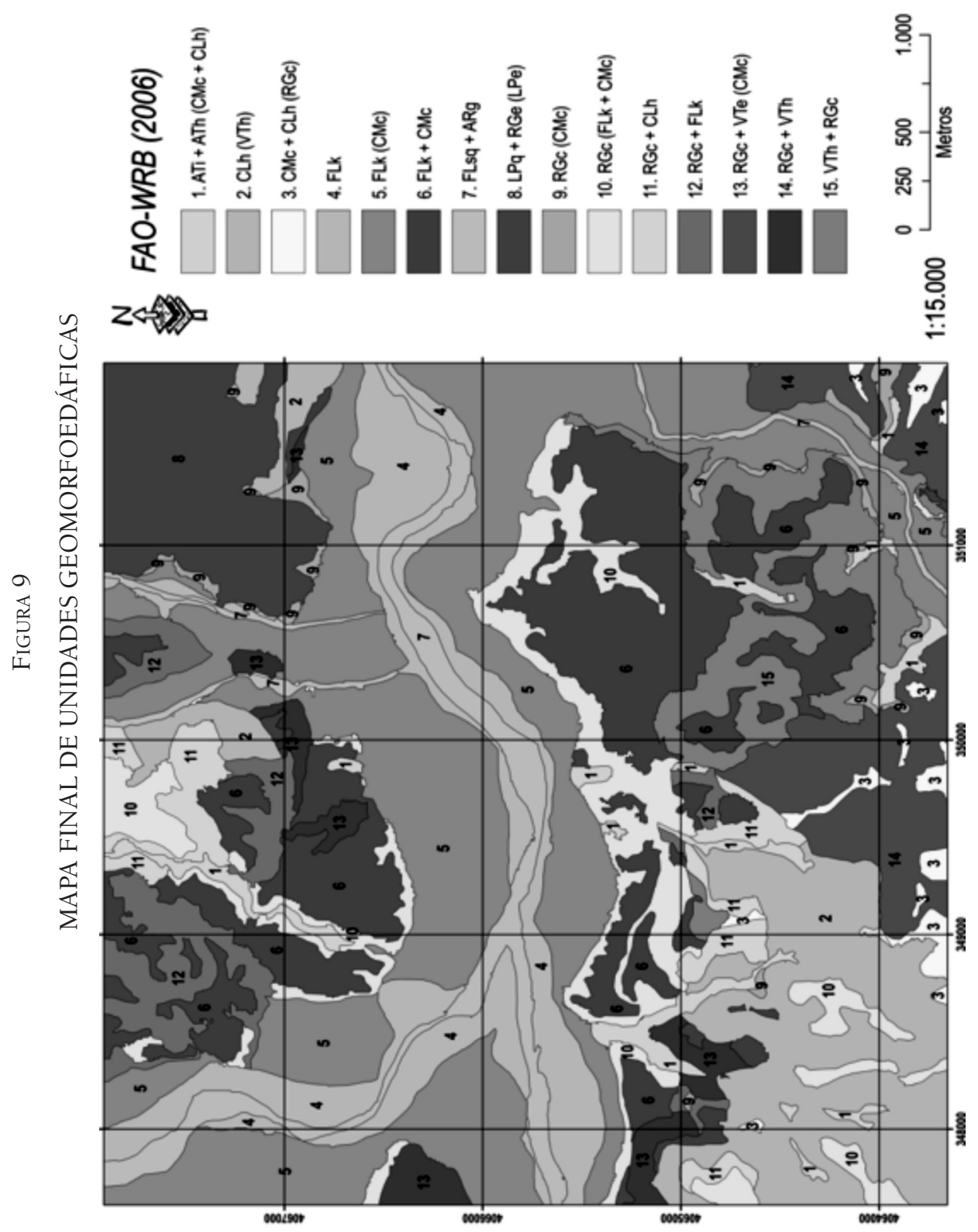

Estudios Geográficos, Vol. LXXVII, 280, pp. 275-310, enero-junio 2016

ISSN: 0014-1496, eISSN: 1988-8546, doi: 10.3989/estgeogr.201610 


\section{Extrapolación de resultados de laboratorio de perfiles de suelos de áreas periféricas}

Para este último paso, se deben estudiar las características de los perfiles del proyecto LUCDEME de las áreas periféricas y seleccionar cuáles serán los utilizados para la extrapolación (tabla 4). Se han incluido un total de quince, teniendo en cuenta su clasificación en la taxonomía de FAO de 1988 y los tipos de suelos principales o más afines a las asociaciones mostradas del mapa geomorfoedáfico anterior.

Se calculan los promedios de cada dato obtenido en el laboratorio por el proyecto LUCDEME, los cuales serán implementados en cada unidad cartografiada en una nueva tabla de atributos en SIG. Como ejemplo, se exponen una serie de mapas significativos de dichas extrapolaciones que no son del todo adecuadas. En cada una se ha superpuesto la silueta del mapa de unidades geomorfoedáficas con el número con el que corresponde en su leyenda, con objeto de comparar sus límites con la propiedad evaluada y el tipo de suelo original.

Para el mapa de clases texturales (figura 10), siguiendo las indicaciones de Porta y Acevedo (2005), los suelos menos permeables, con una mayor compacidad, dificultades para el laboreo y una fuerte energía de retención de aguas serían los relacionados con las texturas arcillosas del cauce principal, y en menor medida, los de las terrazas fluviales y los de los glacis. Por el contrario, los mejores suelos con una textura más adecuada para el laboreo, menos compactos y con menor escorrentía (franco-arenosa, franco-limosa) corresponderían a las unidades de los escarpes y terrazas erosionadas, junto con las laderas irregulares y los taludes del conjunto maláguide. Sin embargo, se aprecian algunas incongruencias en la cartografía: i) los suelos de tipo Fluvisol y Cambisol que en la actualidad permanecen en la actualidad explotados intensivamente no tienen las condiciones texturales, a priori, para serlos; ii) por el contrario, los más susceptibles a la erosión, con problemas de movilización de materiales y de escorrentía superficial son catalogados con mejores aptitudes en este apartado. 
TABLA 4.

PERFILES SELECCIONADOS PARA LA EXTRAPOLACIÓN DE INFORMACIÓN EDAFOLÓGICA

\begin{tabular}{|c|c|c|c|c|c|c|c|}
\hline Hoja & Perfil & $\mathrm{X}$ & $\mathrm{Y}$ & Orden & Suborden & Localización & m.s.n.m. \\
\hline Antequera-1023 & 1 & 339500 & 4100500 & Regosol & Calcárico & $\begin{array}{l}500 \mathrm{~m} \text { al sur del Cortijo } \\
\text { del Cerero }\end{array}$ & 460 \\
\hline Marbella-1065 & 2 & 310800 & 4056400 & Regosol & Calcárico & Al N de la Igualeja & 740 \\
\hline $\begin{array}{l}\text { Málaga } \\
\text { Torremolinos }\end{array}$ & 5 & 368500 & 4069800 & Calcisol & Háplico & $\begin{array}{l}\text { Al SE del Lagar } \\
\text { de los Frailes }\end{array}$ & 320 \\
\hline Vélez Málaga & 6 & 401600 & 4070300 & Cambisol & Calcárico & $\begin{array}{l}\text { Al N del Cortijo } \\
\text { Alberquilla }\end{array}$ & 30 \\
\hline Vélez Málaga & 11 & 420300 & 4068300 & Regosol & Calcárico & $\begin{array}{l}\text { Al N de la Ermita } \\
\text { de San Isidro }\end{array}$ & 30 \\
\hline Álora-1052 & 15 & 353300 & 4075400 & Leptosol & Éutrico & $\begin{array}{l}200 \mathrm{~m} \text { al E de la } \\
\text { Ermita de las Tres Cruces }\end{array}$ & 595 \\
\hline Álora-1052 & 17 & 356600 & 4072700 & Leptosol & Lítico & $\begin{array}{l}600 \mathrm{~m} \text { al SW del } \\
\text { Lagar de los Majones }\end{array}$ & 340 \\
\hline Coín & 25 & 349300 & 4042500 & Cambisol & Crómico & $\begin{array}{l}\text { Entre el Cerro del Pilar y } \\
\text { la Roza del Aguado }\end{array}$ & 80 \\
\hline Coín & 26 & 355700 & 4051600 & Antrosol & Cumúlico & $\begin{array}{l}\text { En las proximidades } \\
\text { de la Casa del Pintor }\end{array}$ & 340 \\
\hline Álora-1052 & 28 & 337200 & 4070600 & Vertisol & Éutrico & $\begin{array}{l}1 \mathrm{~km} \text { al S de } \\
\text { Huerta Nueva }\end{array}$ & 340 \\
\hline Álora-1052 & 29 & 336100 & 4067800 & Regosol & Calcárico & $\begin{array}{l}1 \text { km al NE del } \\
\text { Cerro del Peñón }\end{array}$ & 355 \\
\hline Álora-1052 & 37 & 344400 & 4068500 & Antrosol & Árico & $\begin{array}{l}\text { Ladera E de la } \\
\text { Sierra de Gibralgalia }\end{array}$ & 175 \\
\hline Álora-1052 & 48 & 352900 & 4061600 & Cambisol & Calcárico & $\begin{array}{l}\text { 1,4 km al SW de la cima } \\
\text { de la Sierra de los } \\
\text { Espartales }\end{array}$ & 155 \\
\hline Álora-1052 & 50 & 363400 & 4059600 & Calcisol & Háplico & $\begin{array}{l}300 \mathrm{~m} \text { al W del } \\
\text { Cortijo Blanco }\end{array}$ & 30 \\
\hline Álora-1052 & 52 & 347700 & 4065800 & Fluvisol & Calcárico & $\begin{array}{l}400 \mathrm{~m} \text { al NE de la } \\
\text { Ermita de Casapalma }\end{array}$ & 50 \\
\hline
\end{tabular}

Estudios Geográficos, Vol. LXXVII, 280, pp. 275-310, enero-junio 2016

ISSN: 0014-1496, eISSN: 1988-8546, doi: 10.3989/estgeogr.201610 


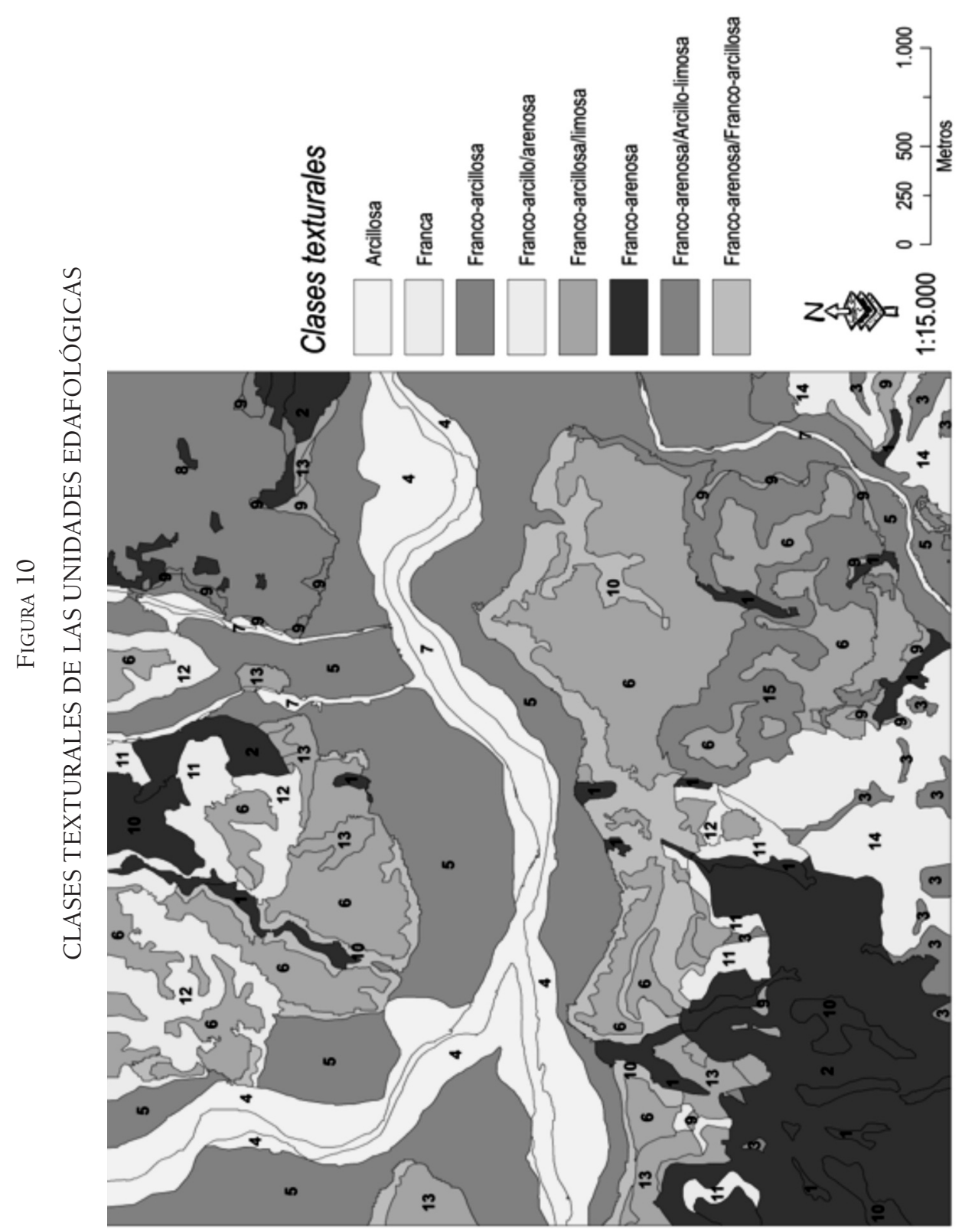

Estudios Geográficos, Vol. LXXVII, 280, pp. 275-310, enero-junio 2016 ISSN: 0014-1496, eISSN: 1988-8546, doi: 10.3989/estgeogr.201610 


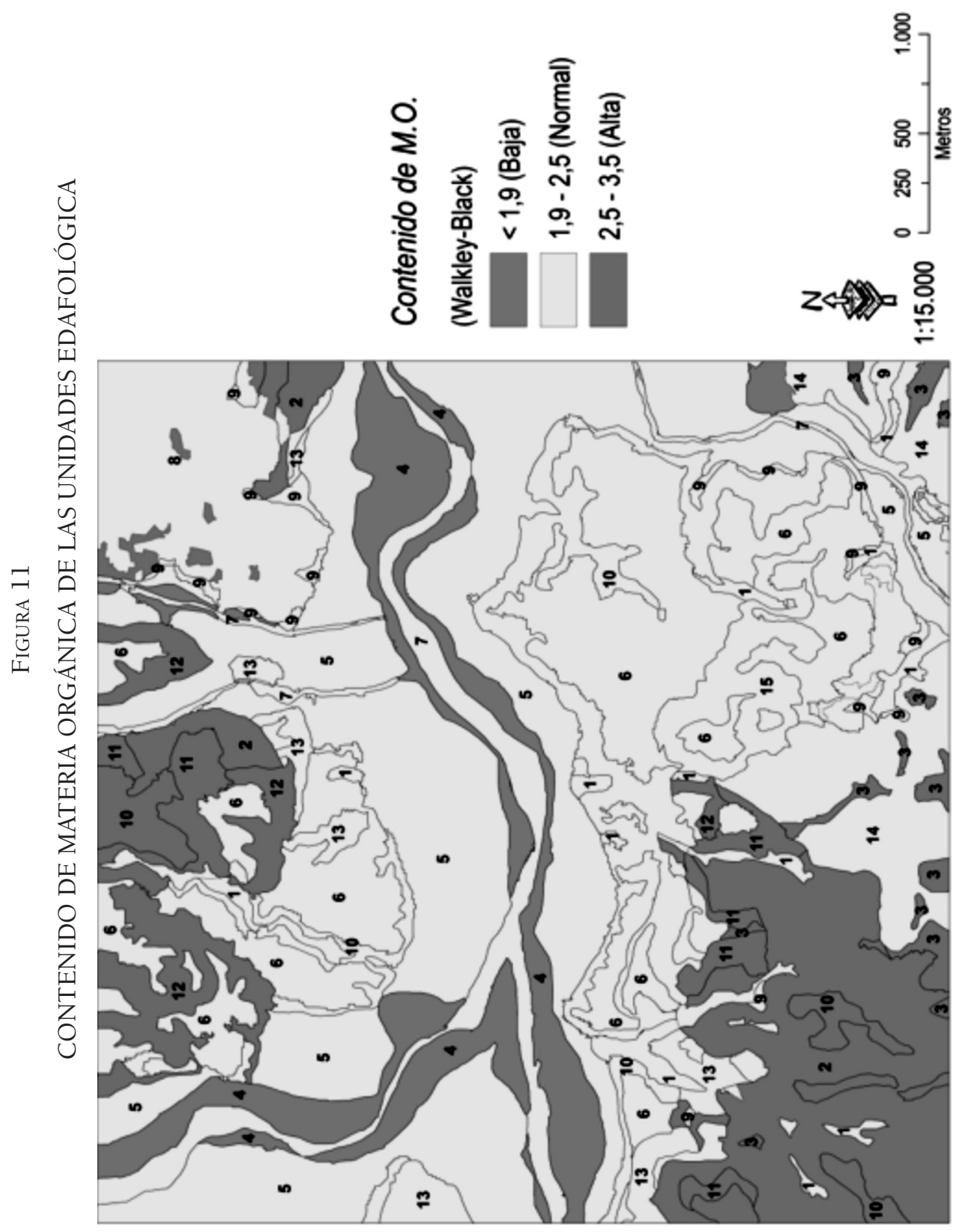

Estudios Geográficos, Vol. LXXVII, 280, pp. 275-310, enero-junio 2016

ISSN: 0014-1496, eISSN: 1988-8546, doi: 10.3989/estgeogr.201610 


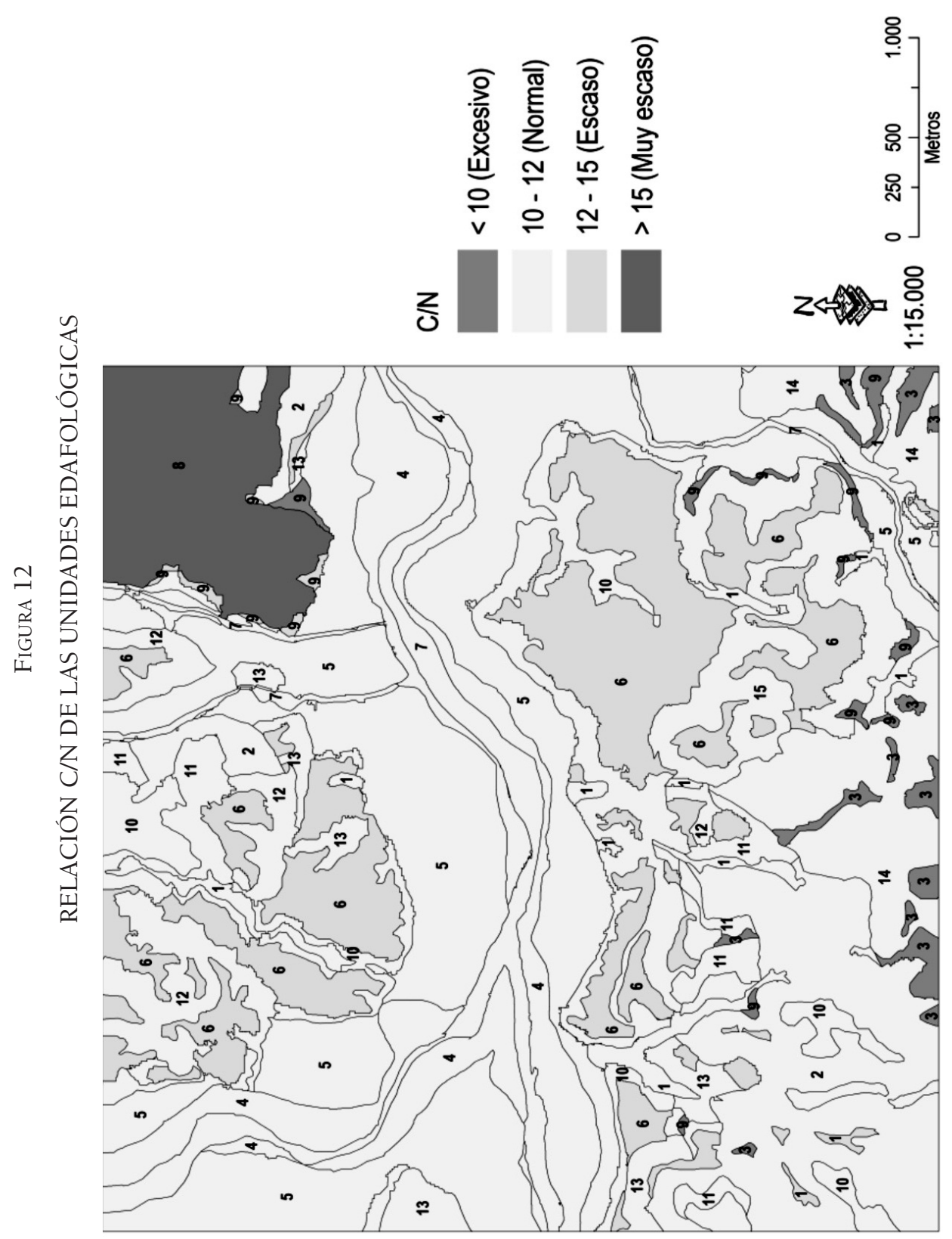

Estudios Geográficos, Vol. LXXVII, 280, pp. 275-310, enero-junio 2016 ISSN: 0014-1496, eISSN: 1988-8546, doi: 10.3989/estgeogr.201610 


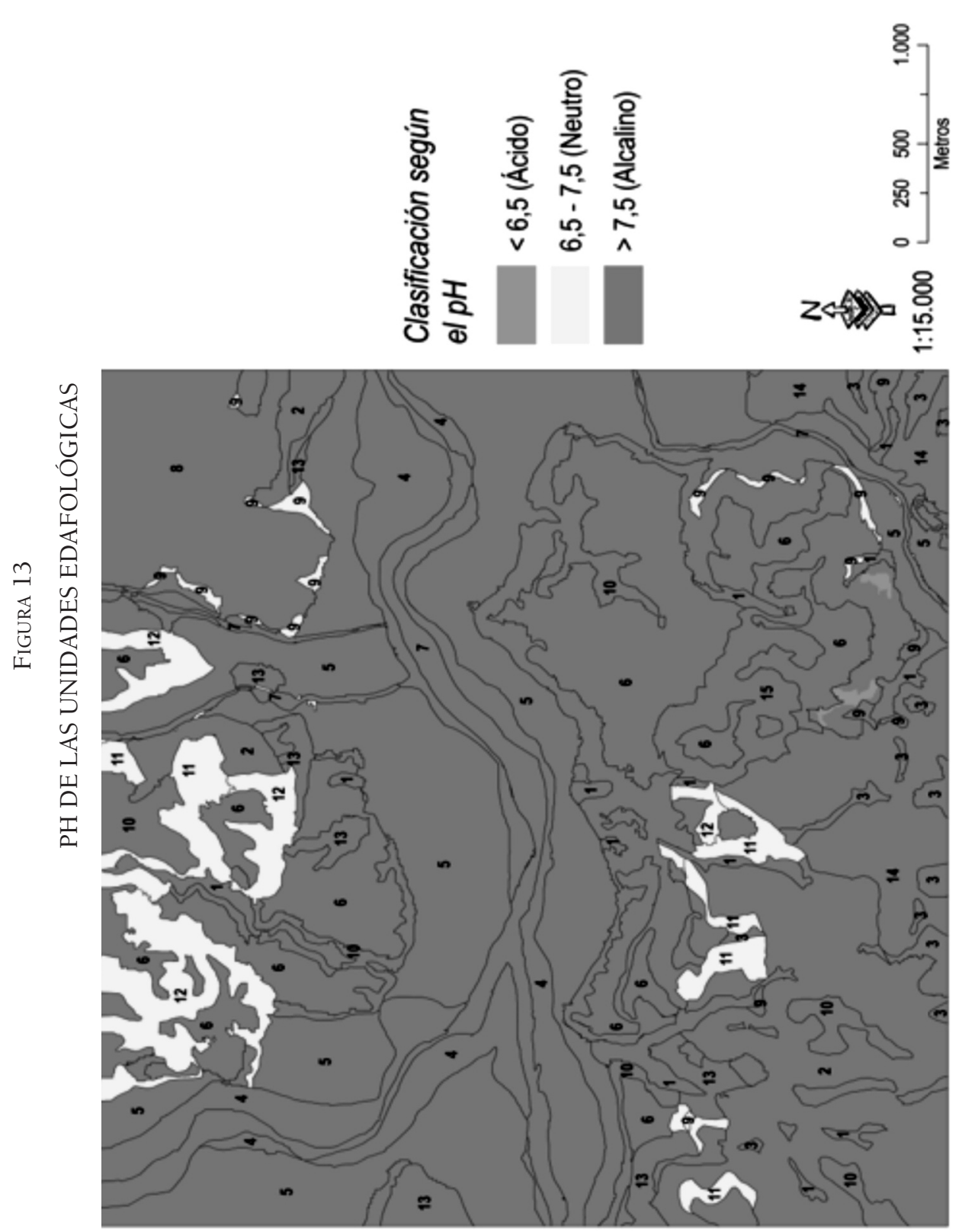

Estudios Geográficos, Vol. LXXVII, 280, pp. 275-310, enero-junio 2016

ISSN: 0014-1496, eISSN: 1988-8546, doi: 10.3989/estgeogr.201610 
Por otra parte, se ha comprobado también en otros mapas de propiedades químicas también significativas (figuras 11, 12, 13, reproducidas en las tres páginas anteriores): contenido de materia orgánica (\%), relación C/N y pH. Se pone en evidencia que las áreas colindantes al río (el lecho estacionalmente inundado y las terrazas fluviales) son cartografiadas como los peores suelos para su explotación agraria o forestal. Así, suelos que en la actualidad albergan bosques de eucaliptos y terrazas fluviales ampliamente cultivadas en la actualidad, aparecen con valores de materia orgánica y liberación de nitrógeno en unos niveles que muestran una baja fertilidad, una mineralización débil y una pobreza en bases que no es lógica, si se tienen en cuenta los niveles generalizados de pH básico en toda el área de estudio. Por el contrario, hacia los taludes, las laderas irregulares y los escarpes, los niveles pasan a estabilizarse de nuevo.

\section{DisCUSIÓN}

En primer lugar, se puede destacar que la metodología utilizada, siendo correcta, puede resultar antigua por la bibliografía en la que se ha basado el estudio (Van Zuidam y Van Zuidam Cancelado, 1979; Dent y Young, 1981; Panizza et al., 1987; Panizza, 2005). Hoy en día, existen publicaciones relevantes que utilizan nuevos métodos y tecnologías para detectar propiedades del suelo relacionadas con la teledetección como el trabajo de Mulder et al. (2011). Este análisis aplica sistemas capaces de georreferenciar particularidades del suelo tras observar la respuesta espectral obtenida. Otras aplicaciones directas combinando otras herramientas para la ordenación del territorio en esta línea son también, por ejemplo, los trabajos de Peter et al. (2014) que utiliza simulaciones de lluvia en climas áridos o Taylor et al. (2009) y Corbane et al. (2012) que discriminan propiedades como la conductividad eléctrica o los componentes superficiales del suelo en campos cultivados con vides.

Sin embargo, a nuestro favor es posible afirmar que: i) dicha metodología, implementando los nuevos sistemas de información geográficos y bases de datos edafológicas, puede convertirse en una actualización del método antiguamente enunciado, por lo que quedaría avanzar incorporando nuevos avances en fotogrametría o modelización; ii) es un procedimiento que no tiene coste alguno, no requiere de medios cartográficos de alta resolución poco asequibles económicamente sin proyectos que lo financien; iii) es fácilmente reproducible en cualquier territorio partiendo de los conocimientos del investigador en técnicas fotointerpretativas y de clasificación de suelos. 
Con respecto a la selección de las variables geomorfológicas utilizadas en este trabajo (morfometría, asociaciones litológicas, morfologías o sistemas de paisaje), sin duda, pueden también ser discutidas. Existe un amplio espectro de posibilidades según los estudios que versan sobre esta temática (Dent y Young, 1981; Peña Monné, 1997; Birkeland, 1984, 1999; Targulian y Goryachkin, 2008); sin embargo, cabría preguntarse realmente: i) cuáles son verdaderamente las variables más correctas para este tipo de estudios edafogeográficos; ii) cuáles serían en este tipo de escala de trabajo y para este medio en concreto (clima mediterráneo, antropización del territorio, gran variedad de litologías y morfologías) las más afines. Debido a esta última cuestión para este estudio, la elección de la morfometría, las morfologías y las asociaciones litológicas fue una elección sencilla por ser las más determinantes es los aspectos geomorfológicos finales del área de estudio. Por otra parte, quizás algo más discutida pueda ser la selección de los sistemas de paisajes (Panizza et al., 1987; Panizza, 2005), tanto por el término que difiere de la definición oficial de "paisaje» (Convenio Europeo del Paisaje de Florencia en el 2000) como por la escala de definición de los elementos cartografiados. Sin embargo, para esta ocasión, se ha preferido seguir las recomendaciones de las variables escogidas por el ITC.

En lo referente a la utilidad de las unidades de diagnóstico de origen geomorfológico y geomorfoedáficas, podemos afirmar que, además de facilitar la clasificación de suelos, también permiten discriminar datos elaborados en el laboratorio que pudieran ser erróneos o, por otra parte, útiles para la investigación agronómica o civil. Por otra parte, son numerosas las posibilidades que aporta para la creación de una base de datos ampliable y actualizada, y que también permita de cara al futuro acceder fácilmente a las potencialidades que un suelo puede aportar en referencia al cambio de uso o permanencia en el mismo.

Por último, en esta línea otro tema de interés a discutir en esta línea sería la conveniencia o no de adjuntar a la tabla final de atributos la extrapolación de datos de perfiles de suelos periféricos. Nuestra conclusión es afín a desestimar esa posibilidad, al menos, en la provincia de Málaga y, más concretamente, en nuestra área de estudio. Esto es atribuido a que: i) existen numerosos errores de identificación de suelos por la parte de la fuente edafológica utilizada en la provincia de Málaga; ii) confiamos en que las características químicas y físicas del suelo son irrepetibles en diferentes contextos espacio-temporales y virtualmente irreproducibles (Keestra et al., 2007; Keestra et al., 2009; Giménez Morera et al., 2010; Zhao et al., 2013; Xiao et al., 2015). Esta línea sería una cuestión importante para profundizar en el futuro como sugieren Liess et al. 
(2012), incorporando herramientas de clasificación de suelos de FAO-WRB como los horizontes de diagnóstico.

\section{CONCLUSIONES}

Este estudio aplicado ha seguido la óptica enunciada por el enfoque de la Edafogeografía que trata de analizar las causas de la distribución espacial del suelo a lo largo del medio.

Las conclusiones obtenidas a partir de este estudio son: i) la combinación del mapa de unidades de diagnóstico de origen geomorfológico y edafológico dan lugar a una cartografía de unidades geomorfoedáficas que facilita la clasificación e identificación de suelos; ii) los mantos edáficos más extendidos en el área de estudio son las asociaciones de Fluvisol cálcico con Cambisol calcárico y Calcisol háplico con Vertisol háplico; iii) la información edafológica de base del proyecto LUCDEME posee una serie de deficiencias y errores que pueden llevar a confusión a la hora de realizar análisis más profundos para la ordenación del territorio; iv) por consiguiente no se recomienda extrapolar valores desde perfiles periféricos a las áreas de estudio; v) se muestra una metodología fácilmente reproducible para otros territorios como herramienta para la creación de futuras bases de datos edafológicas, siempre y cuando se parta de una información de base correcta tras la elaboración de perfiles y análisis de suelos propios.

\section{REFERENCIAS BIBLIOGRÁFICAS}

Aguilar Ruiz, J. (coord.) et al. (1994): Mapas de suelos; hoja 1.052 (Álora); escala 1:100.000 LUCDEME, Granada, Universidad de Granada.

Arnáez, J., Lasanta, T., Ruiz-Flaño, P., Ortigosa, L. (2007): "Factors affecting runoff and erosion under simulated rainfall in Mediterranean vineyards", Soil \& Tillage Research, 93, pp. 324-334, doi: 10.1016/j.still.2006.05.013.

Arnáez, J., Ruiz-Flaño, P., Lasanta, T., Ortigosa, L., Llorente, J. A., Pascual, N., LanaRenault, N. (2012): "Efectos de las rodadas de tractores en la escorrentía y erosión del suelo en laderas cultivadas con viñedos", Cuadernos de Investigación Geográfica, 38, pp. 115-130.

Auerswald, K., Fiener, P. y Dikau, R. (2009): "Rates of sheet and rill erosion in Germany (a meta-analysis)", Geomorphology, 11, pp. 182-193, doi: 10.1016/j.geomorph.2009.04.018.

Birkeland, P. W. (1984): Soils and geomorphology, New York, Oxford University Press. 
Birkeland, P. W. (1999): Soils and geomorphology, New York y Oxford, Oxford University Press. Tercera edición.

Boardman, J., Poesen, J. y Evans, R. (2003): "Socio-economic factors in soil erosion and conservation", Environmental Science Policy, 6, pp. 1-6.

Brevik, E. C., Calzolari, C., Miller, B. A., Pereira, P., Kabala, C., Baumgarten, A. y Jordán, A. (2015): "Soil mapping, classification, and pedologic modeling: History and future directions", Geoderma, 264/B, pp 256-274, doi: 10.1016/j.geoderma.2015.05.017.

Bryan, R. B. (2000): "Soil erodibility and processes of water erosion on hillslope", Geomorphology, 32, pp. 385-415, doi: 10.1007/BF02872682.

Cerdá, A., Gallart, R., Li, J., Papanastasis, V. P., Parmenter, R. R., Turnbull, L., Parsons, A. J. y Wainwright, J. (2013): "Long-range ecogeomorphic processes", en E. N. Mueller, J. Wainwright y A. Parsons, Patterns of Land Degradation in Drylands. Understanding Self-Organized Ecogeomorphic Systems, Sheffield, Springer, pp. 103-139.

Cerdan, O., Govers, G., Le Bissonnais, Y., Oost, K. van, Poesen, J., Saby, N., Gobin, A., Vacca, A., Quinton, J., Auerswald, K., Klik, A., Kwaad, F. J. P. M., Raclot, Damien, Ionita, I., Rejman, J., Rousseva, S., Muxart, T., Roxo, M. J. y Dostal, T. (2010): "Rates and spatial variations of soil erosion in Europe: a study based on erosion plot data", Geomorphology, 122/1-2, pp. 167-177, doi: 10.1016/j.geomorph.2010.06.011.

Chacón Montero, J., Irigaray, C., Lansos, F. y El Hamdouni, R. (2004): Mecánica de suelos y rocas. Prácticas y ensayos, Granada, Área de ingeniería del Terreno, Universidad de Granada.

Corbane, C., Jacob, F., Raclot, D., Albergel, J. y Andrieux, P. (2012): "Multitemporal analysis of hydrological soil surface characteristics using aerial photos: A case study on a Mediterranean vineyard", International Journal of Applied Earth Observation and Geoinformation, 18, pp. 356-367.

Dent D. y Young, A. (1981): Soil Survey and Land Evaluation, Londres, George Allen \& Unwin.

Duchaufour, P. (1987): Manual de Edafología, Barcelona, Masson.

Ferreras, C. y Fidalgo, C. (1991): Biogeografía y Edafogeografía, Madrid, Síntesis. Colección Espacios y Sociedades, 6.

Galacho, F. B. y Arrebola J. A. (2013): "Modelo de evaluación de la capacidad de acogida del territorio con SIG y técnicas de decisión multicriterio respecto a la implantación de edificaciones en espacios rurales", Investigaciones Geográficas, 60/2, pp. 69-85.

García Marín, R., Calvo García-Tornel, F., (2008): "Frecuencia y evolución de rachas secas en la cuenca del Guadalentín (Sureste de España)", Bol. Asoc. Geógrafos Esp., pp. 71-89.

Gebhardt, H., Glaser, R., Radtke, U. y Reuber, P. (2012): Physische Geographie und Humangeographie, Berlin, Spektrum Akademie.

Giménez Morera, A., Ruiz Sinoga, J. D. y Cerdà, A. (2010): "The impact of cotton geotextiles on soil and water losses in Mediterranean rainfed agricultural land", Land Degradation E Development, 21/2, pp. 210-217, doi: 10.1002/ldr.971. 
Goodchild, M. F. (2011): "Scale in GIS: An overview", Geomorphology, 130/1-2, pp. 59, doi: 10.1016/j.geomorph.2010.10.004.

Hengl, T. (2006): "Finding the right pixel size", Computers $E$ Geosciences, 32, pp. 1283-1298, doi: 10.1016/j.cageo.2005.11.008.

Hernández Santana, J.R., López Miguel, C., Méndez Linares, A.P., Bollo Mannet, M., (2010): "Intensidad geomórfica del relieve noroeste del estado de Chiapas, México: un enfoque para la planeación territorial", Cuaternario y Geomorfología, 24, pp. 79-98.

Ibáñez, J. J., Zinck, J. y Jiménez Ballesta, R. (1990): "Soil survey. Old and new challenges", en: J. Zinck (ed.), Soil survey: Perspectives and strategies for the 21st century, Enschede, Países Bajos, Revista del ITC, pp.7-14.

IGME (1978): Mapa geológico. E.1:50.000, Madrid, Mapa Geológico de España. Serie Magna, hoja 1.052.

IUSS Working Group WRB (2006a): Guidelines for constructing small-scale map legends using the WRB, Roma, World Soil Resources, $2^{\circ}$ ed. FAO.

IUSS Working Group WRB (2006b): World Reference Base for Soil Resources 2006 , Roma, World Soil Resources Report 103, FAO.

IUSS Working Group WRB (2007): Land Evaluation. Towards a revised framework, Roma, Land and Water. Discussion paper, $6.2^{\circ} \mathrm{ed}$. FAO.

Keestra, S. D. (2007): "Impact of natural reforestation on floodplain sedimentation in the Dragonja basin, SW Slovenia", Earth Surface Processes and Landforms, 32/1, pp. 49-65, doi: 10.1002/esp.1360.

Keestra, S. D., Bruijnzeel, L. A., Huissteden, J. van (2009): "Meso-scale catchment sediment budgets: combining field surveys and modeling in the Dragonja catchment, southwest Slovenia", Earth Surface Processes and Landforms, 34, pp. 1547 1561, doi: 10.1002/esp.1846.

Kosmas, C., Danalatos, N., Cammeraat, L. H., Chabart, M., Diamantopoulos, J., Farand, R., Gutierrez, L., Jacob, A., Marques, H., Martinez-Fernandez, J., Mizara, A., Moustakas, N., Nicolau, J. M., Oliverso, C., Pinna, G., Puddu, R., Puigdefabregas, J., Roxo, M., Simao, A., Stamou, G., Tomasi, N., Usai, D. y Vacca, A. (1997): "The effect of land use on runoff and soil erosion rates under Mediterranean conditions", Catena, 29, pp. 45-59, doi:10.1016/S0341-8162(96)00062-8.

Lado, M., Paz, A., Ben-Hur, M., (2004): "Organic Matter and Aggregate-Size Interactions in Saturated Hydraulic Conductivity", Soil Sci. Soc. Am. J., 68, 234, doi:10.2136/sssaj2004.2340

Liess, M., Glaser, B. y Huwe, B. (2012): "Making use of the World Reference Base diagnostic horizons for the systematic description of the soil continuum - Application to the tropical mountain soil-landscape of southern Ecuador", Catena, 97, pp. 20-30.

Martínez-Casasnovas, J. A., Ramos, M. C. y Cots-Folch, R. (2010): "Influence of the EU CAP on terrain morphology and vineyard cultivation in the Priorat region of NE Spain”, Land Use Policy, 27, pp. 11-21, doi:10.1016/j.landusepol.2008.01.009. 
Materechera, S. A. (2009): "Tillage and tractor effects on soil compaction in hortocultural fields used for peri-urban agriculture in a semi-arid environment of the North West Province, South Africa", Soil \& Tillage Research, 103, pp. 11-15, doi:10.1016/j.still.2008.09.001. 2009.

Mulder, V. L., De Bruin, S., Schaepman, M. E. y Mayr, T. R. (2011): "The use of remote sensing in oil and terrain mapping. A review", Geoderma, 162, pp. 1-19, doi:10.1016/j.geoderma.2010.12.018.

Natera, J. J. (2007): "Los usos del suelo de la provincia de Málaga”, en J. J. Durán (coord.), Atlas Hidrogeológico de la provincia de Málaga, tomo 2, Madrid, Instituto Geológico y Minero de España y Málaga, Diputación de Málaga, pp. 59-65.

Navarro Pedreño, J., Meléndez Pastor, I. y Gómez Lucas, I. (2012): "Impact of three decades of urban growth on soil resources in Elche (Alicante, Spain)", Spanish Journal of Soil Science, 1/2, pp. 55-69.

Nieves, M. y Torcal, L. (1983): Introducción a la práctica de la fotoedafología, Madrid, INIA (Instituto Nacional de Investigaciones Agrarias).

Ocaña, C. y Larrubia, R. (1993): Agricultura y espacio metropolitano: Málaga y el Bajo Guadalhorce, Málaga, Textos mínimos/Universidad de Málaga.

Oost K. van, Quine, T. A., Govers, G., De Gryze, S., Six, J., Harden, J. W., Ritchie, J. C., McCarty, G. W., Heckrath, G., Kosmas, C., Giraldez, J. V., Da Silva, J. R. y Merckx, R. (2007): "The impact of agricultural soil erosion on the global carbon cycle”, Science, 318, pp. 626-629, doi:10.1126/science.1145724.

Panizza, M. (2005): Manuale di geomorfologia applicata, Roma, FrancoAngeli. Colección Uomo, ambiente, sviluppo.

Panizza, M., Castaldini, D., Bollettinari, G., Carton, A. y Mantovani, F. (1987): "Neotectonic Research in Applied Geomorphologycal Studies", Z. Geomorph. N.F. Suppl., Bd, 63.

Paroissien, J. B., Lagacherie, P. y Le Bissonnais, Y. (2010): “A regional-scale study of multi-decennial erosion of vineyard fields using vine-stock unearthing-burying measurements", Catena, 82, pp. 159-168, doi: 10.1016/j.catena.2010.06.002.

Peña Monné, J. L. (1997): Los mapas geomorfológicos. Características y tipos En: Cartografía geomorfológica básica y aplicada, Zaragoza, Geoforma/Universidad de Zaragoza.

Peter, K. D., d'Oleire-Oltmanns, S., Ries, J. B., Marzolff, I. y Aït Hssaïne, A. (2014): "Soil erosion in gully catchments affected by land-levelling measures in the Souss Basin, Morocco, analyzed by rainfall simulation and UAV remote sensing data", Catena, 113, pp. 24-40, doi:10.1016/j.catena.2013.09.004.

Piccarreta, M., Capolongo, D., Boenzi, F. y Bentivenga, M. (2006): "Implications of decadal changes in precipitation and land use policy to soil erosion in Basilicata, Italy", Catena, 65, pp. 138-151, doi:10.1016/j.catena.2005.11.005.

Poesen, J., Nachtergaele, J., Verstraeten, G. y Valentin, C. (2003): "Gully erosion and environmental change: importance and research needs", Catena, 50, pp. 91-133, doi: 10.1016/S0341-8162(02)00143-1. 
Porta, J. y López Acevedo, M. (2005): Agenda de campo de suelos, Lleida, Mundiprensa/Universidad de Lleida.

Porta, J. y Poch, R. M. (2011): "DPSIR analysis of land and soil degradation in response to changes in land use", Spanish Journal Soil Science, 1, pp. 100-115.

Prasuhn, V. (2011): "Soil erosion in the Swiss midlands: Results of a 10-year field survey", Geomorphology, 126, pp. 32-41, doi: 10.1016/j.geomorph.2010.10.023.

Prosdocimi, M., Jordán, A., Tarolli, P., Keesstra, S., Novara, A. y Cerdà, A. (2016): "The immediate effectiveness of barley straw mulch in reducing soil erodibility and surface runoff generation in Mediterranean vineyards", Sci. Total Environment, 547, pp. 323-330, doi: 10.1016/j.scitotenv.2015.12.076.

Quinton, J. N., Govers, G., Van Oost, K. y Bardgett, R. D. (2010): "The impact of agricultural soil erosion on biogeochemical cycling", Nature Geoscience, 3, pp. 311 314, doi: 10.1038/ngeo838.

Raclot, D., Le Bissonnais, Y., Louchart, X., Andrieux, P., Moussa, R. y Voltz, M. (2009): "Soil tillage and scale effects on erosion from fields to catchment in a Mediterranean vineyard area", Agriculture, Ecosystems and Evironment, 134, pp. 201-210, doi: 10.1016/j.agee.2009.06.019.

Rodrigo Comino, J. (2014): Los suelos de la provincia de Málaga. Revisión y actualización de las fuentes edafológicas según la clasificación de FAO-WRB, Málaga, Publicaciones y Divulgación Científica, Vicerrectorado de Investigación y Transferencia de la Universidad de Málaga.

Rodrigo Comino, J., Brings, C., Lassu, T., Iserloh, T., Senciales González, J. M., Seeger, M., Ruiz Sinoga, J. D. y Ries, J. B. (2015): "Rainfall and human activity impacts on soil losses and rill erosion in vineyards (Ruwer Valley, Germany)", Solid Earth, 6, pp. 823-837, doi: 10.5194/se-6-823-2015.

Rodrigo Comino, J., Ferre Bueno, E. y Senciales González, J. M. (2014): “Evaluación de la peligrosidad con criterios geomorfológicos a través de las unidades de diagnóstico en el territorio de Casapalma (Valle de Guadalhorce, España)", Anales de Geografía, 34/2, pp. 163-183, doi.org/10.5209/rev_AGUC.2014.v34.n2.47076.

Rodrigo Comino, J., Iserloh, T., Morvan, X., Malam Issa, O., Naisse, C., Keesstra, S. D., Cerdà, A., Prosdocimi, M., Arnáez, J., Lasanta, T., Ramos, M. C., Marqués, M. J., Ruiz Colmenero, M., Bienes, R., Ruiz Sinoga, J. D., Seeger, M. y Ries, J. B. (2016): "Soil Erosion Processes in European Vineyards: A Qualitative Comparison of Rainfall Simulation Measurements in Germany, Spain and France", Hydrology, 3, p. 6, doi: 10.3390/hydrology3010006.

Rodrigo Comino, J. y Senciales, J. M. (2013): "La Edafogeografía. La quinta rama olvidada de la Geografía Física", Cuadernos Geográficos, 52, pp. 1-23.

Rodrigo Comino, J., Senciales, J. M. y Ferre Bueno, E. (2014): “Análisis de los cambios de usos del suelo en el área de Casapalma (Valle del Guadalhorce, Málaga) entre 1991 y 2007", Papeles de Geografía de la Universidad de Murcia, 59-60, pp. 157-171.

Ruiz-Sinoga, J. D. y Martínea-Murillo, J. F. (2009): "Effects of soil surface components on soil hydrological behaviour in a dry Mediterranean environment 
(Southern Spain)", Geomorphology, 108, pp. 234-245, doi: 10.1016/j.geomorph. 2009.01.012.

Ruiz-Sinoga, J. D., Romero-Díaz, A., Ferre-Bueno, E. y Martínez-Murillo, J. F. (2010): "The role of soil surface conditions in regulating runoff and erosion processes on a metamorphic hillslope (Southern Spain): Soil surface conditions, runoff and erosion in Southern Spain", Catena, 80/2, pp. 131-139, doi: 10.1016/j.catena.2009.09.007.

Rukhovich, D. I., Wagner, V. B., Vil'chevskaya, E. V., Kalinina, N. V. y Koroleva, P. V. (2011): "Problems of using digitized thematic maps on the territory of the former soviet union upon the creation of the 'Soils of Russia' geographic information system", Eurasian Soil Science, 44/9, pp. 957-968, doi: 10.1134/S1064229311090110.

Sanz de Galdeano, C. y López Garrido, A. C. (2013): "Tectónica de las sierras penibéticas de Abdalajís y de Huma (provincia de Málaga, España). Su relación con el contacto con la Zona Interna", Estudios Geológicos, 69/2, pp. 133-147.

Soja, E. W. (2001): Postmetropolis. Critical Studies of cities and regions. Oxford, Blackwell.

Targulian, V. O. y Goryachkin, S. V. (2008): Soil Memory Soil as a Memory of BiospherGeosphere-Antroposphere Interactions, Moscú, . URSS.

Taylor, J. A., Coulouma, G., Lagacherie, P. y Tisseyre, B. (2009): "Mapping soil units within a vineyard using statistics associated with high-resolution apparent soil electrical conductivity data and factorial discriminant analysis", Geoderma, 153, pp. 278-284. doi:10.1016/j.geoderma.2009.08.014.

USDA (2010): Keys to Soil Taxonomy, Washington, DC, USDA-Natural Resources Conservation Service. Décimo primera edición.

Viguera Rubio, J., Albarrán, Á., Llera, F., Ferrera, E. y García, T. (2004): Estudio de suelos y su analítica, Badajoz, @becedario/Instituto de Ciencias de la Educación (I.C.E.).

Wang, K., Zhang, C. y Weidong, L. (2013): "Predictive mapping of soil total nitrogen at a regional scale: A comparison between geographically weighted regression and cokriging", Applied Geography, 42, pp. 73-85, doi: 10.1016/j.apgeog.2013.04.002.

Xiao, L., Hu, Y., Greenwood, P. y Kuhn, N. J. A (2015): “Combined Raindrop Aggregate Destruction Test-Settling Tube (RADT-ST) Approach to Identify the Settling Velocity of Sediment", Hydrology, 2, pp. 176-192, doi: 10.3390/hydrology2040176.

Yaalon, D. H. (1975): "Conceptual models in pedogenesis. Can the soils farming functions be solved?", Geoderma, 14, pp. 189-205.

Zhao, G., Mu, X., Wen, Z., Wang, F. y Gao, P. (2013): "Soil erosion, conservation, and Eco-enviroment changes in the Loess Plateau of China", Land Degradation \& Development, 24, pp. 499-510, doi: 10.1002/ldr.2246.

Zinck, J. A. (1988): Soil Survey Courses, Enschede, Países Bajos, ITC.

Zinck, J. A. (2012): Geopedología: Elementos de geomorfología para estudios de suelos y de riesgos naturales, Enschede Países Bajos, ITC.

Zuidam, R. A. van y Zuidam Cancelado, F. I. van (1979): Terrain analysis and classification using aerial photographs. A geomorphologycal approach Enschede, Enschede, 


\section{SITIOS WEBS}

Ministerio de Agricultura, Alimentación y Medio Ambiente. Disponible en: http://www.magrama.gob.es (Fecha de consulta: 04/2014).

European Commission (área Environment). Disponible en: http://ec.europa.eu/environment/soil/index_en.htm (Fecha de consulta: 04/2014).

e-SOTER Project. Disponible en: http://www.esoter.net/ (Fecha de consulta: 04/2014).

Global Soil Map. Disponible en: ttp://www.globalsoilmap.net/ (Fecha de consulta: 04/2014).

Fecha de recepción: 30 de abril de 2014.

Fecha de aceptación: 18 de septiembre de 2015.

\section{RESUMEN}

Se presenta un estudio sobre suelos desde un punto de vista edafogeográfico, cuya área experimental está localizada en un medio mediterráneo dentro de un territorio tradicionalmente agrícola como el Valle del Guadalhorce en la provincia de Málaga (España). La geografía del suelo es una disciplina que pretende estudiar las causas de la distribución de los tipos de mantos edáficos a lo largo del medio y su relación con el ser humano. Para dicho fin, en este trabajo se parte de la elaboración de un mapa de unidades de diagnóstico de origen geomorfológico del ITC (Faculty of Geo-Information Science and Earth Observation, Enschede, Países Bajos) y la cartografía edafológica del proyecto LUCDEME. Tras reescalar y homogeneizar dicha información de partida, se obtiene un mapa final de unidades geomorfoedáficas donde las asociaciones predominantes son: Fluvisol cálcico con Cambisol calcárico y Calcisol háplico con Vertisol háplico. Al no existir perfiles con información de las propiedades físicas y químicas para todas las asociaciones edafológicas obtenidas, se ha extrapolado dichos análisis desde resultados a partir de datos de perfiles periféricos $(\mathrm{pH}$, materia orgánica, textura y relación C/N). Con esta acción, se demuestra que la cartografía resultante no es recomendable para su uso como herramienta para la ordenación del territorio.

Palabras Clave: Edafogeografía; ordenación del territorio; SIG; unidades de diagnóstico; unidades geomorfoedáficas.

\section{ABSTRACT}

The present work shows a study about soils, which are located in a Mediterranean traditional agricultural area (Guadalhorce Valley in Málaga, Spain) from an edaphogeographical point of view. The soil geography pretends to analyze the causes of the distribution of the kinds of soils along the environment and their relationships with humans. For this purpose, a combined methodology of ITC (Faculty of Geo-Informa- 
tion Science and Earth Observation, Enschede, The Netherland) with approaches to the Geomorphology (geomorphological diagnostics units) and the pedological cartography of LUCDEME project have been used. After rescaling and homogenizing this information, a geomorphoedaphological unit map was elaborated. The most predominant pedological associations were classified as: Calcic Fluvisol with Calcaric Cambisol and Haplic Calcisol with Haplic Vertisol. For all the obtained pedological associations, there were any physical and chemical laboratory analyses; therefore properties from peripheral soil profiles were extrapolated. Through the final mappings about $\mathrm{pH}$, organic matter, texture and $\mathrm{C} / \mathrm{N}$ were noted that these extrapolations are not recommendable for the land management.

KEY WORDS: Edaphogeography; land management; GIS; diagnostics units; geomorphoedaphical units. 